\title{
(Bio)conjugation Strategies Applied to Fluorescent Semiconductor Quantum Dots
}

\author{
Goreti Pereira, ${ }^{a, b}$ Camila A. P. Monteiro, ${ }^{a, c}$ Gabriela M. Albuquerque, ${ }^{a, b}$ \\ Maria I. A. Pereira, ${ }^{a, c}$ Mariana P. Cabrera, ${ }^{a, b}$ Paulo E. Cabral Filho, ${ }^{a, c}$ \\ Giovannia A. L. Pereira, ${ }^{a, b}$ Adriana Fontes ${ }^{a, c}$ and Beate S. Santos ${ }^{\circledR} *, a, d$ \\ ${ }^{a}$ Grupo de Pesquisa em Nanotecnologia Biomédica (NanoBio), \\ Universidade Federal de Pernambuco, Recife-PE, Brazil \\ ${ }^{b}$ Departamento de Química Fundamental, Universidade Federal de Pernambuco, \\ 50740-540 Recife-PE, Brazil \\ ${ }^{c}$ Departamento de Biofísica e Radiobiologia, Universidade Federal de Pernambuco, \\ 50670-901 Recife-PE, Brazil \\ ${ }^{d}$ Departamento de Ciências Farmacêuticas, Universidade Federal de Pernambuco, \\ 50740-560 Recife-PE, Brazil
}

\begin{abstract}
Quantum dots (QDs) are semiconductor nanocrystals, which present unique photophysical properties, enabling their application as new fluorescent platforms for biomedical sciences. Colloidal QDs are end-capped with organic or inorganic compounds, not only to prevent their agglomeration but also to provide reaction sites for the attachment of targeting (bio)molecules, nanoparticles or other interfaces, for specific biological purposes. The (bio)conjugation can involve non-covalent or covalent interactions, which can be accomplished through different strategies. The final assembly needs to maintain its chemical and optical stability and biochemical functionality. Although a relative good comprehension of the experimental procedures has been established, the bioconjugation process is still a challenge. The present manuscript aims to review the main (bio)conjugation strategies successfully applied to QDs, describing the steps necessary to prepare stable targeting fluorescent nanoplatforms, as well as some usual methods used to evaluate and optimize this process.
\end{abstract}

Keywords: nanocrystals, fluorescence, cross-linkers, conjugation strategies

\section{Introduction}

Nanotechnology is a field that has been attracting the interest of many research groups and, nanomaterials have become key elements for science and technology, especially due to their physicochemical properties that can be controlled according to their size and/or shape. A class of emerging nanomaterials is the quantum dots (QDs), fluorescent semiconductor nanocrystals with diameters from about 2 to $10 \mathrm{~nm}$. These nanostructures have proved to be a powerful tool to develop electroluminescent devices, optical switching systems, photovoltaic cells, and more recently biomedical nanoprobes. ${ }^{1,2}$ The "discovery" of QDs was ascribed to Alexei Ekimov, a Russian physicist that synthesized, for the first time (in 1981),

*e-mail: beate.saegesser@gmail.com
$\mathrm{CuCl}_{2}$ semiconductor microcrystals in a glass matrix. ${ }^{3}$ Another important Russian researcher was Alexander Efros, who established the first theoretical principles for QDs, i.e., he explained the optical behavior of this special class of semiconductors by electron confinement. These findings lead Ekimov and Efros, ${ }^{4}$ in 1985, to study quantum size effects in semiconductors. The authors observed an appreciable variation in the QDs wavelength emission to low values when their size was decreased. Concurrently and motivated by these discoveries, the American chemist Louis Brus synthesized the first colloidal suspensions of CdS QDs. ${ }^{5}$ Nevertheless, when QDs were first studied, researchers did not expect that this class of nanomaterials would be one day useful for biological applications. However, in 1998, two independent studies presenting the use of QDs for biological purposes were published. ${ }^{6,7}$ 
The first bioapplications reported in the literature were essentially performed applying colloidal CdSe QDs synthesized by hydrophobic routes, which needs further preparation procedures to render a biocompatible material. Currently, a great number of different hydrophobic or hydrophilic procedures to obtain QDs are already well established. To guarantee the colloidal stability, preventing agglomeration and precipitation during the nanocrystals' growth, stabilizers and surfactant agents need to be associated to the surface of the nanoparticles. This coating layer controls the growth of the nanocrystals and can also serve to functionalize them, facilitating their conjugation with other molecules, nanoparticles, or other interfaces. For biological applications, the end-capped QD's surface, in general, consists of alkyl thiol molecules such as mercaptosuccinic acid and cysteamine or polymers like polyethylene glycol (PEG). Also, a variety of nanocrystals compositions can be found, consisting mainly of atoms of the II-VI, III-V or IV-VI groups of the periodic table..$^{1,2}$ Good examples are composed of binary combinations of these atoms forming nanocrystals, such as CdTe or ZnSe QDs (II-VI group), InP QDs (III-V group), or PbSe
QDs (IV-VI group). ${ }^{8,9}$ Lately, new types of copper-based ternary and quaternary multicomponent QDs have also been prepared, as they have more environmental friendly compositions and show near-infrared emission profiles. Examples of ternary and quaternary nanocrystals include $\mathrm{Cu}-\mathrm{In}-\mathrm{S}$ (CIS), $\mathrm{Cu}-\mathrm{In}-\mathrm{Se}$ (CISe), $\mathrm{Cu}-\mathrm{Zn}-\mathrm{In}-\mathrm{S}$ (CZIS), $\mathrm{Zn}-\mathrm{Ag}-\mathrm{In}-\mathrm{Se}$ (ZAISe) and $\mathrm{Zn}-\mathrm{Ag}-\mathrm{In}-\mathrm{S}$ (ZAIS). ${ }^{10-12}$

The QDs' fluorescence arises from the electron-hole recombination of excited electrons that were promoted to the conduction band (CB) and return to the valence band (VB). As a consequence of the quantum confinement, the nanocrystals will present energy level discretization and bandgap energy (Eg) inversely proportional to their size, as shown in Figure 1a. ${ }^{13}$ Thus, the quantum confinement influences directly the QDs optical properties, providing size tunability of the emission band, exemplified by the naked-eye fluorescence of different sized CdTe QDs shown in Figure $1 b .1,2,13$ The QDs emission wavelength is also inversely proportional to the value of Eg: the higher Eg the lower the emission wavelength. Furthermore, QDs have a high surface area, due to their reduced size, which results in a large number of surface defects that affect their photoluminescence (a)

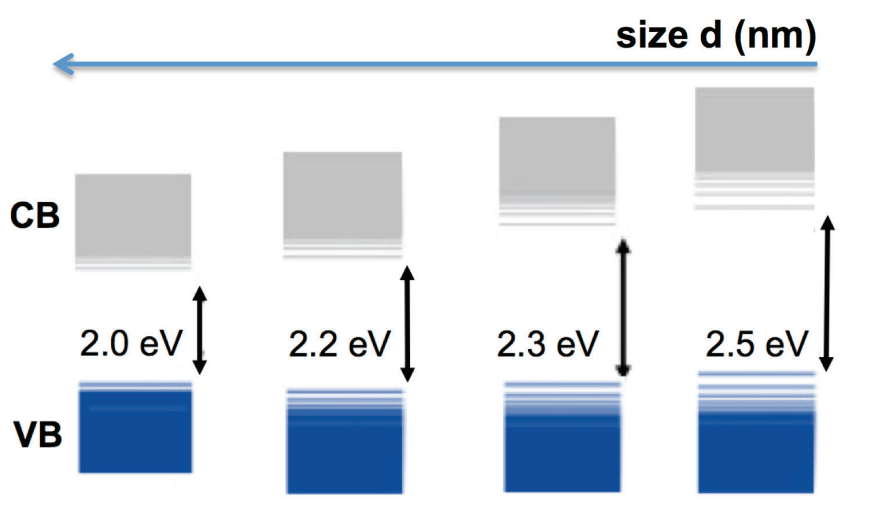

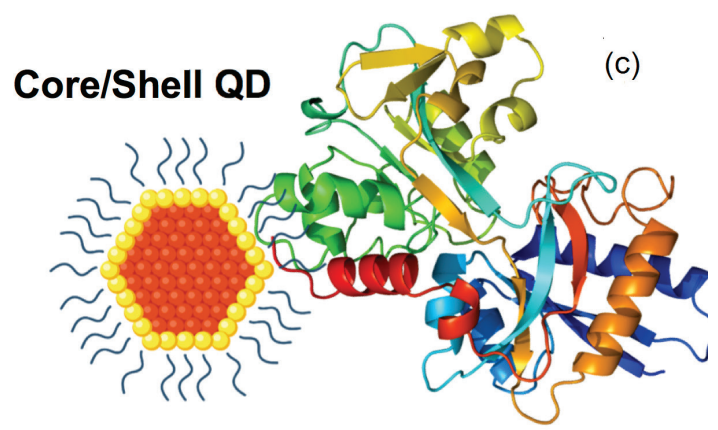

Biomolecule

(b)

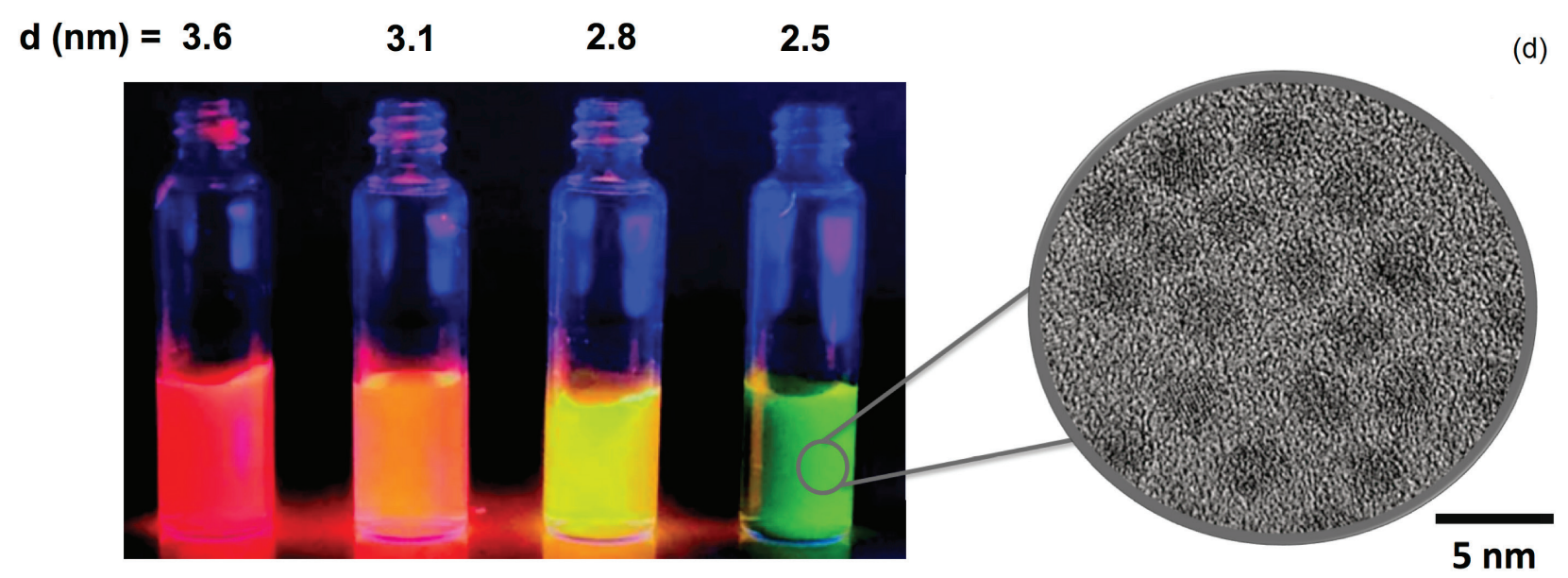

Figure 1. (a) Schematic representations of the quantum confinement effects; (b) CdTe QDs size-tunable emission; (c) QDs core/shell structure for biological application, and (d) a TEM image of the nanocrystals. QDs images from NanoBio research group. 
properties. Thus, in order to reduce the nonradiative recombination sites, the nanocrystals are usually coated with a second semiconductor layer, usually of wider Eg, that passivates the surface, improving the luminescence efficiency and resulting in a core/shell structure (Figure 1c). ${ }^{1,2,9,13}$ A representative transmission electron microscopy (TEM) of the nanocrystals is also presented in Figure 1d.

For core/shell QDs structures: (i) the core is responsible for the emission; (ii) the shell avoids non-radiative transitions, and (iii) the surface molecules confer colloidal stability and functional groups to be used for further conjugation to molecules of biological interest (Figure 1c). The association of QDs and biomolecules can be carried out by covalent, adsorption or other types of conjugations. For QDs prepared in aqueous media, the surface molecules also confer charges to the nanocrystals' surface. For instance, carboxylate coated nanocrystals will be negatively charged at physiological $\mathrm{pH}$, whereas the amino-functionalized ones will be cationic. PEG molecules present a zwitterionic character, which is also important for biological interactions..$^{14-16}$

Over the years, it was revealed that, besides the size-tunable emission, QDs present many other special features for biological application purposes: (i) broad absorption and narrow emission spectra, allowing the use of only one light source to obtain a multicolor labeling; (ii) high quantum yields, providing bright fluorescent images; (iii) low photobleaching rates allowing the follow-up of long-term real-time processes, and (iv) highly active surfaces for conjugations. ${ }^{2,17-19}$

In recent years, in vitro and in vivo QD biological applications have remarkably increased due to the outstanding physicochemical properties of these nanocrystals. For that, QDs have been conjugated with a variety of molecules, such as nucleic acids,,$^{20}$ antibodies and/or their fragments, ${ }^{21,22}$ lectins ${ }^{23,24}$ and carbohydrates. ${ }^{24,25}$ The QDs associated with specific molecules have been applied, as fluorescent nanotools, in a great variety of studies, such as quantitative and qualitative cell labeling, ${ }^{22}$ receptor expression and recycling investigation, ${ }^{26}$ yeast cells labeling, ${ }^{23}$ parasite metabolism monitoring, ${ }^{27}$ in vivo animal applications, ${ }^{28,29}$ cancer diagnosis and therapy. ${ }^{30}$ In addition, several studies have been developed in the field of fluoroimmunoassays and biosensors, ${ }^{1,31,32}$ exploiting both optical and semiconductor properties of QDs. ${ }^{33-35}$ Also, more recently, QDs have been associated with paramagnetic compounds to develop multimodal nanoprobes for optical and magnetic resonance imaging. ${ }^{36-38}$ Furthermore, QDs were also applied as photosensitizers in photodynamic therapy. ${ }^{39,40}$ To be successful in all of these applications it is fundamental to prepare an efficient and stable bioconjugated system. Since the first successful trials, that applied semiconductor QDs as fluorescent biolabeling tools in 1998, a large number of different conjugating approaches were tested. The first procedures consisted of adaptations of known conjugation techniques developed to link organic fluorescent molecules with proteins and other molecules and species. Currently, we observe a large number of different conjugation approaches described in the literature, which take into account (i) the nature of the QDs (i.e., size, hydrophobicity, chemical coating); (ii) the chemical system that will be conjugated and (iii) the final biological or chemical target we aim to apply. Thus, the present manuscript aims to describe the state of the art of the conjugation strategies applied to semiconductor QDs and the most relevant methods for conjugation evaluation.

\section{Bioconjugation Strategies}

Conjugation of QDs to molecules for biological applications was first described by two independent groups, at the same time, in 1998. Bruchez et al. ${ }^{6}$ conjugated QDs to biotin, to label F-actin cell filaments using a biotinstreptavidin strategy. Simultaneously, Chan and $\mathrm{Nie}^{7}$ conjugated QDs to two proteins (IgG and transferrin-Tf) using a carbodiimide (ethyl-3-(dimethylaminopropyl) carbodiimide, EDC or EDAC) as the coupling agent. Since these breakthroughs, conjugation of fluorescent nanocrystals has grown exponentially, and many other conjugation strategies have been developed. 2,16,41,42

In general, during a bioconjugation process, it is necessary to take into account the following observations: (i) the bioconjugation procedure cannot affect the biomolecule intrinsic activity; (ii) the procedure cannot quench the nanoprobe optical properties; (iii) the quantity of the biomolecules on the QDs' surface should be controlled; (iv) the obtained bioconjugate should be stable under physiological conditions; $(v)$ the bioconjugate cannot establish unspecific bindings with biological systems and therefore, when needed, blocking the unreacted coupling agents should be applied.

To associate QDs with other compounds, such as (bio) molecules, nanoparticles (NPs), and surfaces (from now on referred to as biomolecules in the text), there are two main approaches: non-covalent binding and covalent linking (as represented in Figure 2). Non-covalent bindings can be accomplished by different types of interactions between the nanocrystals and the biomolecules, such as by adsorption or chemical affinity. Covalent binding require that a covalent bond is formed between the QDs' coating agent and the biomolecule, and many coupling agents can be used to accomplish this. ${ }^{42,43}$ Here, we describe the majority of the 


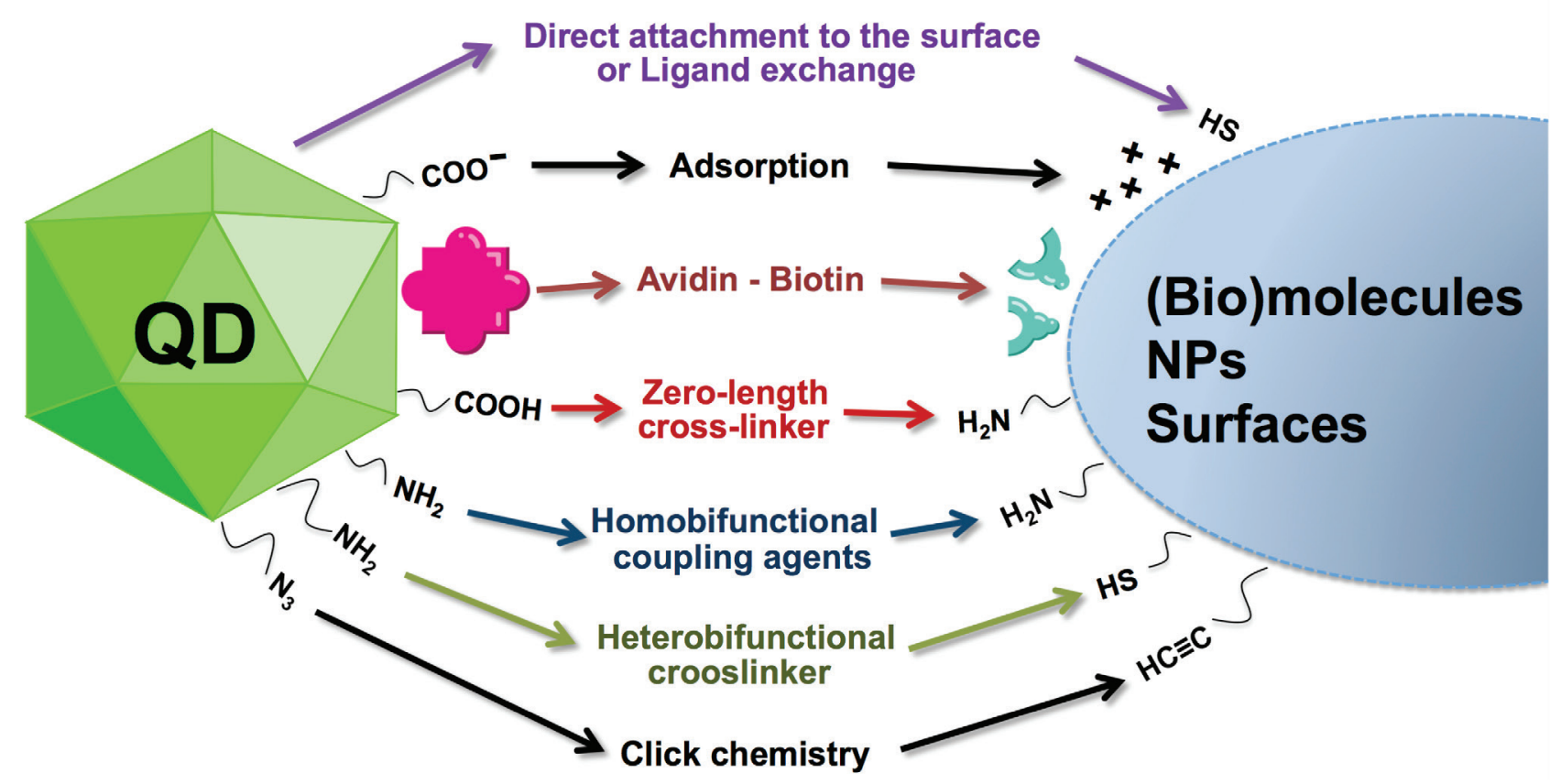

Figure 2. Main strategies currently applied for QDs' conjugation to either (bio)molecules, nanoparticles (NPs) or surfaces.

non-covalent and covalent strategies currently applied to associate QDs with molecules, surfaces or other particles. We present the chemical principles behind each conjugation method and some well succeeded examples from the literature.

\subsection{Non-covalent binding strategies}

Non-covalent binding between nanocrystals and biomolecules can be achieved by different methods, such as adsorption, direct attachment to the QDs' surface and (strept)avidin-biotin affinity.

\subsubsection{Adsorption}

A common and simple strategy for QDs' bioconjugation is the passive adsorption, which has been extensively used to associate QDs with biomolecules and other small molecules. This method may be used with either hydrophobic or hydrophilic QDs. The adsorption of biomolecules on hydrophobic NPs is a result of strong interactions between the non-polar regions or aromatic amino acids with the NPs' surface coating. On the other hand, hydrophilic NPs can establish electrostatic interactions between their surface coatings and polar molecules. Proteins usually possess a hydrophilic surface and a hydrophobic center, thus to interact with non-polar particles a conformational change must occur. These conformational changes can lead to protein denaturation and loss of biological activity, so adsorption by electrostatic interactions is generally the method of choice. . $^{16,42,43}$
Electrostatic adsorption relies on the attraction between species with opposite charges, originating a non-specific bond between the NP and the biomolecule. A frequently used strategy for QDs' non-covalent conjugation is the association of negatively-charged QDs with positivelycharged biomolecules (Scheme 1). The formed bond is, in general, weak and dependent on the magnitude of the QDs' and the biomolecule' charge. It is also susceptible to medium conditions, such as $\mathrm{pH}$, ionic strength and temperature. Furthermore, under certain conditions and in the presence of competitive molecules (i.e., other biomolecules present in the biological environment), a dissociation followed by an exchange with another molecule can occur. ${ }^{17,42,43}$

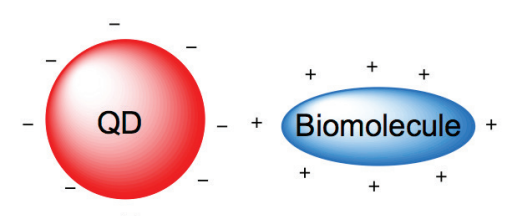

Scheme 1. Schematic representation of electrostatic adsorption of negatively-charged QDs and positively-charged biomolecules.

This type of bioconjugation was applied to conjugate QDs with a wide range of biomolecules, mostly proteins, ${ }^{44,45}$ porphyrins,${ }^{40}$ lectins,${ }^{23,46}$ carbohydrates ${ }^{47}$ and nucleic acids. ${ }^{48}$

For example, Viana et al. ${ }^{40}$ produced systems consisting of mercaptosuccinic acid (MSA) functionalized CdTe QDs bounded electrostatically with $\mathrm{Zn}^{\text {II }}$ porphyrins ( $\left.\mathrm{ZnP}\right)$. Porphyrin derivatives are widely used in photodynamic 
therapy presenting great efficiency in reactive oxygen species (ROS) production. The electrostatic interaction was obtained by mixing QDs and $\mathrm{ZnP}$ molecules at different ratios, exploring the negative and positive charge interactions of CdTe-MSA and ZnP, respectively. The conjugation efficiency was evaluated by absorption and emission spectroscopies, as well as, zeta potential measurements.

The same strategy was used in 2017 by Cunha et al. ${ }^{23}$ to conjugate negatively-charged QDs with Cramoll lectin, for Candida albicans labeling (Figure 3). For the preparation of conjugates, the $\mathrm{pH}$ of the hydrophilic CdTe QDs was adjusted to 7.0, followed by the addition of Cramoll, at $25^{\circ} \mathrm{C}$. This $\mathrm{pH}$ allowed an efficient and specific cell labeling, around $92 \%$, determined by flow cytometry.

\subsubsection{Direct attachment to the surface}

Biomolecules can be attached directly on QDs' surface by dative interactions, usually by thiol interactions or metal-affinity coordination. A dative bond is a coordination bond, formed by the donation of two electrons by a single atom of the biomolecule. These bonds have lower energies and higher lengths than covalent bonds. Thus, they are sensitive to $\mathrm{pH}$, oxidation, and exchange with similar molecules. ${ }^{17,42,43}$

(a)

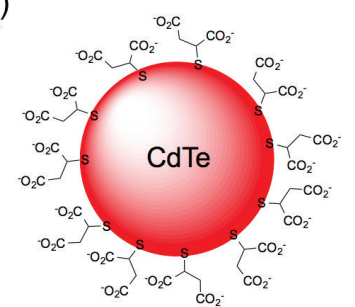

CdTe-COOH QD
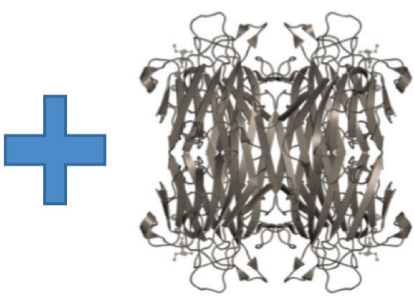

Cramoll Lectin
Metal-affinity interactions between QDs and biomolecules are usually accomplished by the binding of the imidazole ring, present at histidine (His) residues, to the cationic metal on the QDs' surface (Scheme 2). Increasing numbers of His amino acids in the biomolecule will increase affinity to the QDs, improving the bioconjugation. ${ }^{42,43,49}$

Thiolated molecules have also been used in non-covalent conjugation of QDs (Scheme 3). The sulfhydryl group can establish dative bonds with the metal present on the QDs' surface. The main drawback of this strategy is that the biomolecule needs to have available thiol residues. Another concerning issue is that the QDs' surface capping agents cannot hinder the thiol binding by competing for the available surface sites. ${ }^{42,43,49}$

The association of QDs and biomolecules by direct attachment to the nanocrystal surface has been widely used, for example with proteins, ${ }^{50-52}$ carbodydrates, ${ }^{25,53}$ polymers $^{54}$ and viruses. ${ }^{55}$

In search of more efficient bimodal contrast agents for optical and magnetic resonance imaging (MRI), McAdams et al. ${ }^{37}$ anchored Gd ${ }^{I I I}$ complexes on the QDs surface. The diethylenetriaminepentaacetic (DTPA) dianhydride was reacted with 4-aminothiophenol, followed by complexation with $\mathrm{Gd}^{\mathrm{III}}$, yielding the thiol-functionalized DTPA-Gd ${ }^{\text {III }}$ chelates. These chelates were then attached to

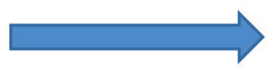

(b)

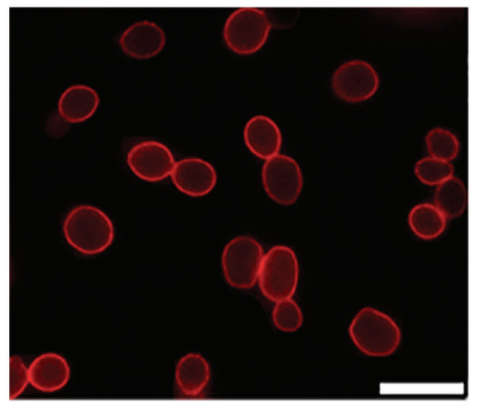

Figure 3. (a) Schematic representation of the conjugation of CdTe-COOH QDs with Cramoll lectin by adsorption. (b) Microscopy image (scale bar: $10 \mu \mathrm{m}$ ) of C. albicans yeasts labeled with QD-Cramoll bioconjugate (adapted from reference 23 with copyright permission 2018 from Elsevier).

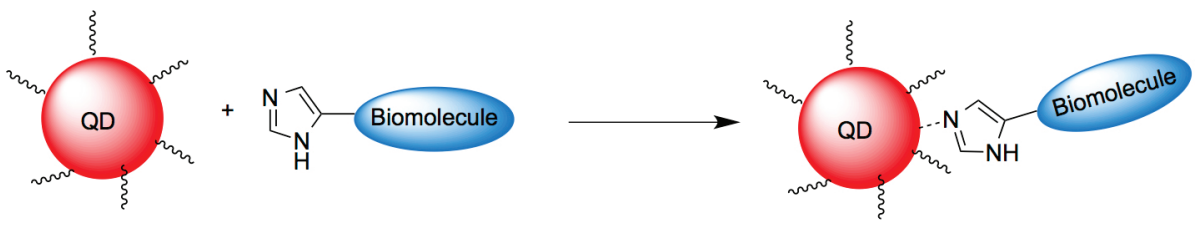

Scheme 2. Schematic representation of a non-covalent conjugation of QDs to biomolecules by metal-affinity interactions.

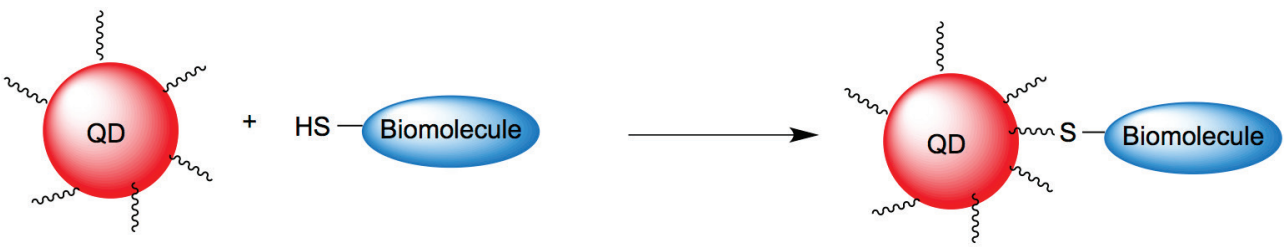

Scheme 3. Schematic representation of a non-covalent conjugation of QDs to biomolecules by thiol binding. 
the surface of hydrophobic CdSe/ZnS QDs by a ligand exchange methodology. The conjugates were characterized by TEM, energy dispersive X-ray spectroscopy (EDS) and UV-Vis spectroscopy.

In a similar approach, polyhistidine linkers were used by Palomo et al. ${ }^{25}$ to associate QDs to cell glycans. In this work, designed peptides containing a polyhistidine linker were coupled to sialic acid residues and, then attached to the surface of hydrophobic CdSe/ZnS QDs. The conjugate efficiency was evaluated by electrophoresis, and its specificity was confirmed by fluorescent studies applying these probes to target Sambucus nigra lectin.

\subsection{3. (Strept)avidin-biotin binding}

The (strept)avidin-biotin complex has been widely used to conjugate several molecules and particles (Scheme 4a). This method is based on the natural high-affinity interactions between avidin or streptavidin and biotin, which are similar to that of receptor-ligand or enzyme-substrate interactions. However, the (strept)avidin-biotin binding is one of the strongest non-covalent interactions known, approaching the covalent bond strength, and for this reason, the process is often described as a covalent conjugation method. The strength of (strept)avidin-biotin complex is extremely useful in bioconjugate chemistry since it is resistant to changes in $\mathrm{pH}$, buffer salts, temperature and, procedure manipulations, such as multiple washings steps. ${ }^{43,56,57}$

Biotin is a small molecule (Scheme $4 b$ ), also known as vitamin $\mathrm{H}$ or $\mathrm{B} 7$ that can be inserted in biomolecules or particles without affecting their activity or properties. This molecule possesses a carboxylic acid, which allows the covalent conjugation to a variety of species. ${ }^{43,56}$

Avidin is a glycoprotein constituted by four identical subunits, each one containing one binding site for biotin. The binding pocket, in each subunit, possesses tryptophan and lysine amino acids and mannose and $\mathrm{N}$-acetylglucosamine carbohydrates. Due to its composition, avidin has a great tendency for non-specific binding with other molecules. Furthermore, its high positive charge can activate unwanted electrostatic interactions with negatively-charged cell membranes. The removal of avidin carbohydrate residues by a chemical deglycosylation procedure has been used to overcome this drawback, without affecting the avidin affinity. ${ }^{43,56}$

(a)

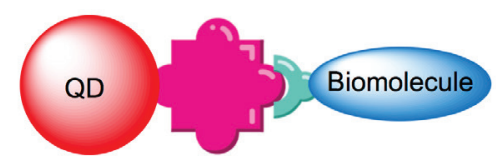

Streptavidin is a biotin-binding protein, similar to avidin, containing also four binding sites to biotin, one in each subunit. The main difference is that streptavidin is nonglycosylated protein, therefore with reduced tendency to bind to carbohydrate receptors. The commercially available streptavidin is a lower-mass form of the native protein, having a molecular mass lower than avidin. The overall charge of streptavidin is also lower than that of avidin, which makes it less likely to interact electrostatically with other molecules or cell membranes. For this reason, this protein is also less soluble in aqueous medium than avidin. ${ }^{43,56}$

The (strept)avidin-biotin conjugation has often been used, however, this strategy presents a major drawback which is related to the large size of the final structure since strept(avidin) is a protein and most of the targeted biomolecules are also proteins. This limitation makes this approach less popular than the carbodiimide coupling strategy. ${ }^{42,57}$ Another issue is that biomolecules need to be biotinylated. As this step is usually achieved by linking the biotin to the biomolecule using a spacer, the length of this spacer may affect the (strept)avidin-biotin binding rate. ${ }^{43}$

For bioconjugating QDs using this method, a frequent approach is the functionalization of their surfaces with streptavidin or avidin and the use of biotinylated biomolecules. The tetrameric structure of (strept)avidin, which allows the binding of more than one biotin group, can be especially used to enhance the desired QD signal. ${ }^{43,56,58}$ Several groups have reported the use of this protocol to conjugate QDs to peptides, ${ }^{31,59}$ proteins, ${ }^{60}$ antibodies ${ }^{61,62}$ and nucleotides. ${ }^{63,64}$

The method of streptavidin-biotin bonding was applied by Ghimire et al. ${ }^{65}$ to develop transparent thin films involving QDs conjugated to polymers. Initially, the chitosan-polystyrene (CS-PS) copolymer was reacted with biotin and then added to streptavidin-conjugated CdSe/ZnS QDs. This methodology allows a uniform distribution of conjugated QDs in the film, maintaining their original optical properties.

Tang et al. ${ }^{31}$ used QDs as fluorescent labels to detect the amyloid-beta peptide 1-42 $\left(\mathrm{A} \beta_{1-42}\right)$, a biomarker for Alzheimer disease. Using microplates, these authors prepared a double antibody sandwich immunoassay. Firstly, a specific C-terminal antibody was immobilized in the plate and the free sites blocked by a protein-free blocking buffer. Then the biotinylated antibody of A $\beta 1-42$

(b)

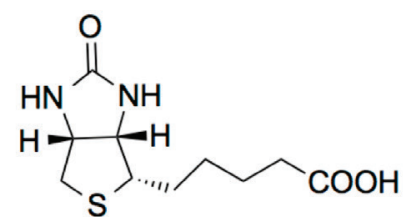

Scheme 4. (a) Schematic representation of QDs functionalized with avidin targeting biotynilated biomolecules; (b) molecular structure of biotin. 
was added, followed by the addition of commercial $\mathrm{CdSe} / \mathrm{ZnS}$-streptavidin QDs. The results showed that this sandwich immunoassay was able to detect Alzheimer's peptides, even in a complex biological fluid sample.

\subsection{Covalent linking}

Covalent linking between QDs and molecules or other particles can be achieved using different types of coupling agents. These cross-linkers have been divided into three classes: zero-length, homobifunctional and heterobifunctional. The zero-length coupling agents mediate the reaction between two functional groups, forming a new bond without additional atoms (no spacer is added between the two original molecules). Homobifunctional agents possess two identical reactive sites, one at each end of a spacer arm (usually an alkyl chain), allowing for the conjugation between two identical functional groups. Heterobifunctional cross-linkers have two different reactive sites that can establish a covalent bond with two distinct functional groups. Both, homobifunctional and heterobifunctional coupling agents possess a spacer arm between the reactive sites, which will be added between the two-conjugated species during the linking process. ${ }^{43}$

The coupling reagent choice needs to take into account the functional groups present either on QDs' surface or in the molecule. Here, we present different covalent coupling strategies, pointing the main coupling agents used for QDs bioconjugation, organized by functional groups present in the species involved in the conjugation process.

\subsubsection{Carboxylic acid-amine coupling}

The carbodiimide method is a standard procedure to link covalently amino acids and has been widely used in peptide synthesis. Carbodiimides are zero-length coupling agents, which mediate the reaction of carboxylic acids and amines, forming amide bonds (Scheme 5a). Carbodiimides derivatives can be water-soluble or water-insoluble. For QDs conjugation the water-soluble carbodiimides are the chosen ones, allowing the reaction in aqueous buffer solutions. Among the water-soluble carbodiimides, EDC (Scheme $5 b$ ) is frequently applied along with an additive, usually $N$-hydroxysuccinimide (NHS) or sulfo-NHS (an NHS water-soluble compound). . $^{16,43,66}$

The reaction occurs via the nucleophilic attack of the oxygen of the carboxylic acid to the carbodiimide, forming an $O$-acylisourea, a highly reactive intermediate, which can react with amines to form an amide bond. The addition of NHS allows the formation of a second intermediate, more soluble and stable, that finally reacts with the amine yielding the final product. The first intermediate formed $(\mathrm{O}$-acylisourea) reacts slowly with amines and hydrolysis can occur, reducing the conjugation yield. Another limitation associated with this low reaction rate is the potential racemization of the amino acids, which can lead to protein denaturation or activity loss. The second intermediate, formed in the reaction with NHS, is stable to hydrolysis in water and reacts rapidly with amines, creating a stable amide bond (Scheme 6). The ideal $\mathrm{pH}$ range for the EDC coupling is 4.5-7.5, beyond this $\mathrm{pH}$ value the reaction rate and yield decrease. ${ }^{43,66}$

Biological systems can possess amine groups that could bind to activated carboxylic groups, so a final

(a)
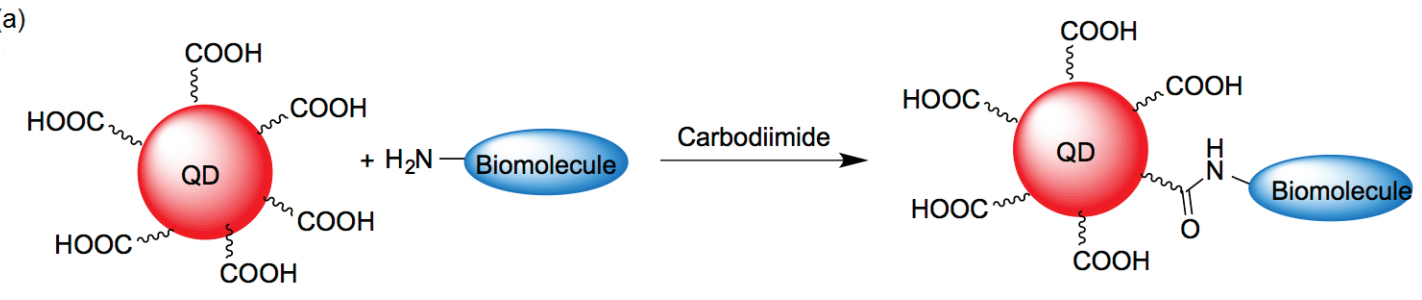

(b)

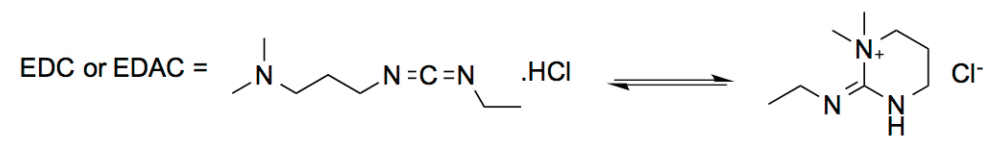

Scheme 5. (a) Schematic representation of QDs conjugation to biomolecules by a carbodiimide strategy; (b) structure of the water-soluble carbodiimide EDC.

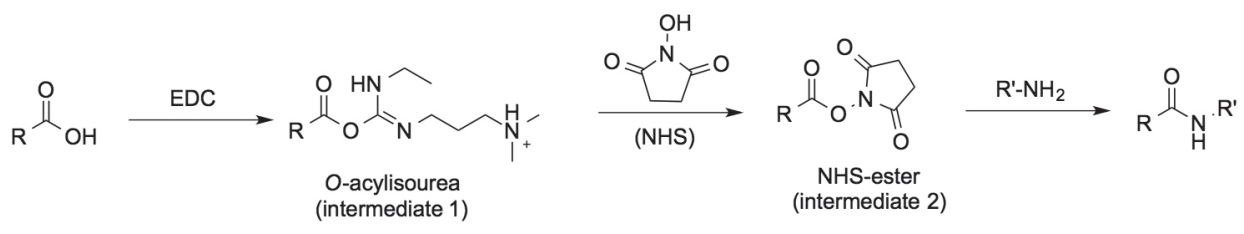

Scheme 6. Reaction steps between a carboxylic acid and an amine using EDC and NHS as coupling agents. 
step is required to block the unreacted NHS-esters. This could be performed by reacting the bioconjugate with glycine or tris(hydroxymethyl)aminomethane (TRIS). The carbodiimide activation will decrease the nanoparticles surface negative charge, which could lead to aggregation. This limitation can be minimized by reducing the amount of coupling agents used. ${ }^{43,67}$

The major drawback for this methodology is the potential polymerization of the biomolecules, which typically contain several amine and carboxylic groups. This issue could require a purification step, which may not be simple, efficient or could lead to the biomolecule denaturation. To overcome this limitation carboxyliccoated QDs are incubated with EDC/sulfo-NHS, and the active ester form is then mixed with the biomolecule or an amine-containing molecule. This two-step protocol gives greater control over the conjugation process, decreasing the biomolecules polymerization. ${ }^{43,57}$

Nevertheless, this carbodiimide strategy has been the method of choice for many research groups that need to conjugate QDs to biomolecules, ${ }^{23,26,68}$ and also to other nanoparticles $^{36,69}$ and small molecules. ${ }^{70}$

Cabral Filho et al. ${ }^{26}$ employed fluorescent techniques to better understand cancer cell biology through the evaluation of cell surface receptors for transferrin. The authors conjugated Tf with hydrophilic CdTe-MSA QDs, using the coupling reagents EDC and Sulfo-NHS. The conjugation proceeded at $\mathrm{pH}$ ca. 5.5 and with a ratio of QDs:Tf 1:2, forming the QDs-Tf probes. The bioconjugation efficiency of the QDs-Tf nanosystems was confirmed applying the fluorescence microplate assay (FMA). Specific labeling tests were performed using HeLa cells as a model. Also, QDs-Tf conjugates allowed for the investigation of Tf receptors in human glioblastoma cell lines (U87 and DBTRG-05MG), using confocal microscopy and flow cytometry (Figure 4). The results showed that DBTRG-05MG cell line presented high staining when compared to U87 cells, indicating that DBTRG-05MG cells expressed and internalized a higher amount of Tf receptors (Figure 4).

Another application of this bioconjugation technique, described by the same research group, is the preparation of promising bimodal MRI and fluorescent probes composed of CdTe QDs and ferrofluid (FF) magnetic NPs. ${ }^{36}$ To activate the carboxyl-coated CdTe-MSA QDs, EDC and sulfo-NHS were added in sequence. Then, FF NPs functionalized with 3-aminopropyltriethoxysilane (APTES) were inserted into the QDs suspension. The steps of surface modification were followed by zeta potential measurements and spectral analysis.

Zhang et al. ${ }^{71}$ employed ZnS QDs doped with $\mathrm{Mn}^{\mathrm{II}}$ associated with dopamine (Dopa-Mn:ZnS QDs) to detect tyrosinase (TYR) activity in chicken serum. The TYR activity was detected based on the fluorescence signal reduction, demonstrating the potential to apply this probe for TYR identification activity in living cells. The bioconjugation of dopamine with QDs was based on the
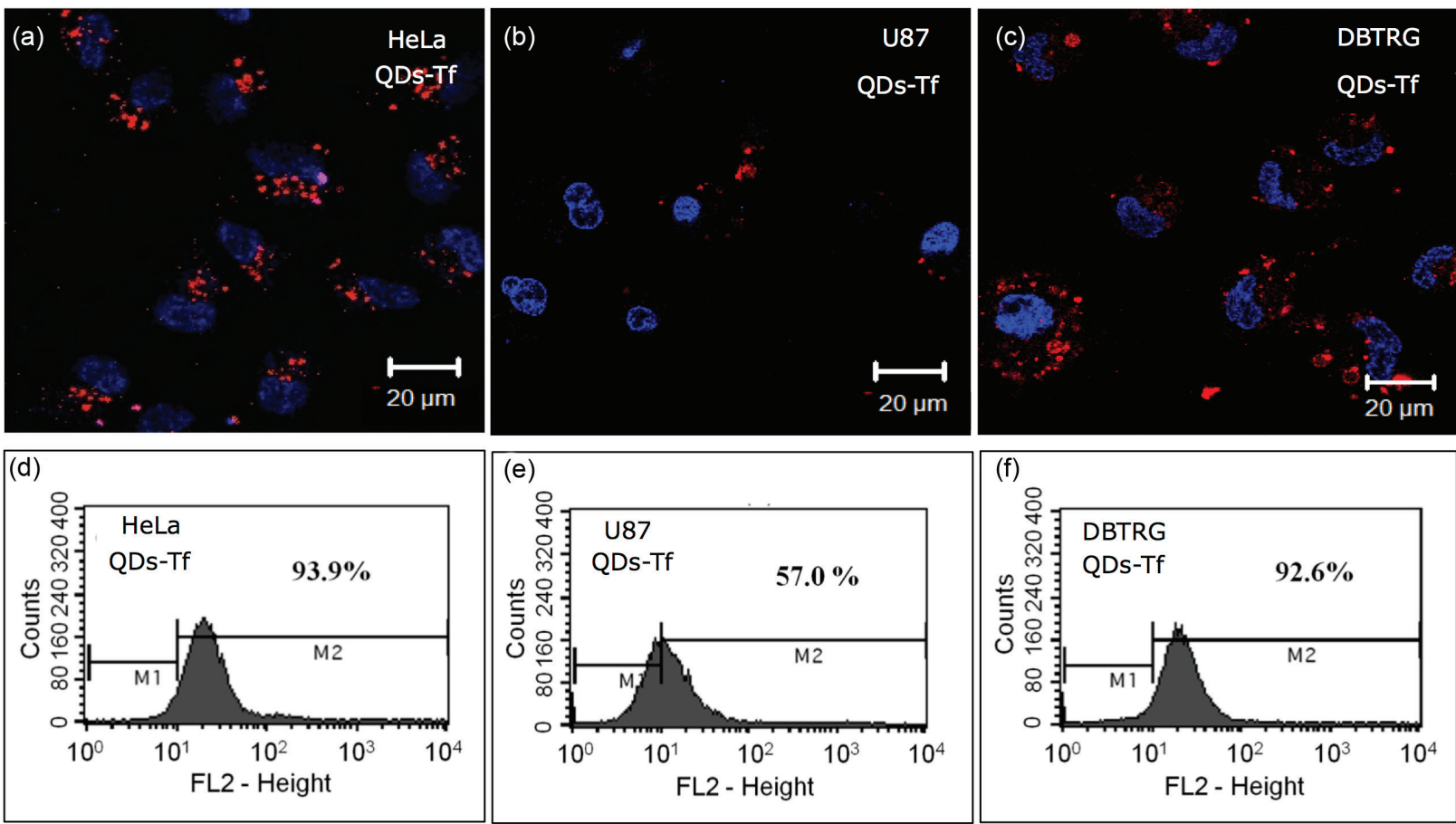

Figure 4. Fluorescence microscopy images and flow cytometry results of HeLa (a and d), U87 (b and e) and DBTRG-05MG (c and f) cell lines labeled with QDs covalently conjugated with transferrin (adapted from reference 26 with copyright permission 2016 from Elsevier). 
EDC/NHS method, which explores the primary amine groups of dopamine and the carboxyl groups of QDs.

One more study, realized by Yu et al. ${ }^{32}$ presented a fluorescence-linked immunosorbent assay (FLISA) for identification of deoxyribonucleic acid (DNA) methyltransferase 1 (DNMT 1), whose activity is related to some types of tumors. The bioconjugates were used in FLISA to detect free DNMT1 and showed potential to indicate DNMT1 levels in serum samples. In this work, DNMT1 polyclonal antibody ( $\mathrm{PcAb})$ was conjugated with $\mathrm{CdSe} / \mathrm{ZnS}$ QDs by EDC/NHS. The conjugates were characterized by TEM and microplate assays.

\subsubsection{Amine-amine coupling}

Two amines, one on the QD surface and the other one on the biomolecule, can be covalently conjugated using homobifunctional-coupling agents, which contain two identical amine-reactive sites, such as two aldehydes or two NHS esters. Homobifunctional reagents can be used in one- or two-step protocols, both procedures presenting some issues. In one-step protocols, the QDs, coupling agents and the biomolecules are mixed together at the same time. Since each amine-reactive site can react either with QDs or with biomolecules, cross-linking between two QDs or two biomolecules can also occur. Two-step methods imply the addition of the coupling agent to the QDs, in a first step, followed by purification to remove the coupling agent excess, to form an activated intermediate. Then, in a second step, the biomolecule is added, originating the final bioconjugate. The potential problem for this approach is the hydrolysis (in aqueous medium) of the activated intermediate, preventing the second reaction with the biomolecule. ${ }^{43}$

Nucleophiles, such as amines and alcohols, can be added to aldehydes using mild conditions. The reaction can be either catalyzed by acid or base, being more efficient at low $\mathrm{pH}$ or high $\mathrm{pH}$ conditions. The nucleophilic addition of primary amines to aldehydes produces a Schiff base.
The addition of the amine to the carbonyl groups forms a tetrahedral intermediate which, by proton transfer from nitrogen to oxygen, produces an aminoalcohol. The oxygen is then protonated, a water molecule is removed and, after proton transfer to water, an imine is yielded (Scheme 7). The Schiff base is not very stable, but it can be stabilized by reduction with sodium borohydride. ${ }^{43}$ The nucleophilic addition of the alcohol oxygen to the carbonyl of an aldehyde is also possible and generates a hemiacetal.

This conjugation strategy has been used to associate biomolecules containing amine groups to aminated QDs, usually utilizing glutaraldehyde, a molecule containing two aldehyde moieties. One aldehyde group forms a Schiff base with the amino groups of the QDs, and the other aldehyde groups form identical bonds with the amino groups of the biomolecules (Scheme 8). Nevertheless, the resulting Schiff base is not stable and this linkage can be reversible. Reactions with glutaraldehyde are favored in alkaline medium, being more efficient at high $\mathrm{pH}^{43}$

Another approach to conjugate covalently two amines is a homobifunctional-coupling agent containing the NHS ester at each end of its spacer arm. These amine-reactive groups originate a stable amide when reacting with amines (Scheme 9). A popular reagent used in QDs bioconjugation is bis(sulfosuccinimidyl)suberate $\left(\mathrm{BS}^{3}\right)$, which contains eight carbon atoms in its spacer chain, linking the two NHS esters and, the sulfonyl group that makes this reagent aqueous soluble (Scheme 9). Optimal conditions for this reaction requires a pH between 6.5 to $8.5 .{ }^{43}$

As mentioned above this strategy can be carried out in a one- or two-step procedure, and both present some disadvantages. In the single-step reaction procedure, all reagents are mixed together, providing a low control of the conjugation process that could lead to a huge mixture of products, such as polymerization of the biomolecules. The two-step method presents a higher control of the cross-linking process, decreasing the polymerization problem, however, the activated intermediate formed in

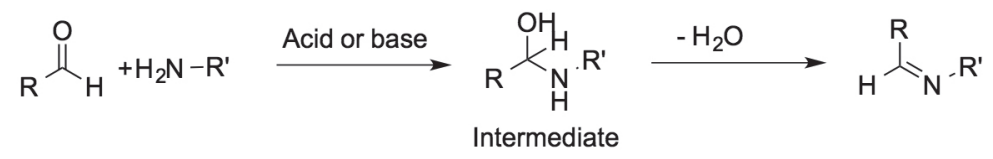

Scheme 7. Reaction between an aldehyde an amine.

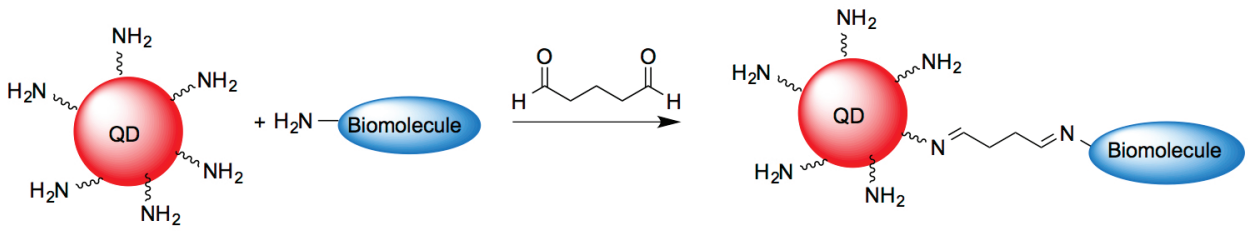

Scheme 8. Schematic representation of the QDs conjugation to biomolecules using glutaraldehyde. 


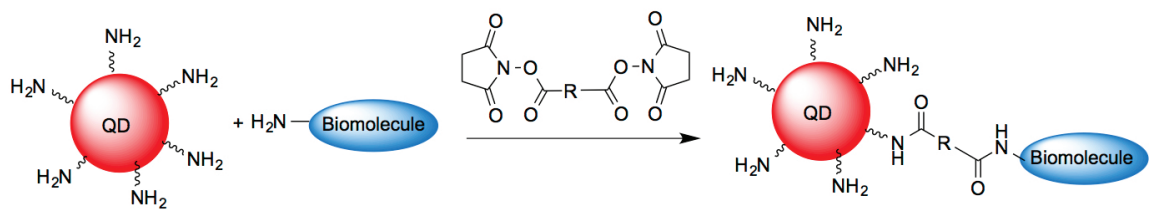

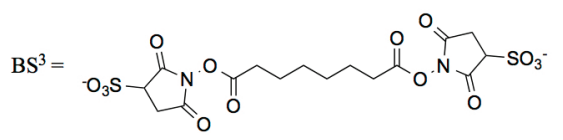

Scheme 9. Schematic representation of QDs conjugation to biomolecules by an amine-amine binding and structure of the $\mathrm{BS}^{3}$ coupling agent.

the first step could suffer hydrolysis in aqueous solution. Another issue for this strategy is the presence of amino groups in biological systems, which could lead to an undesired link between the nanoparticle and its target. ${ }^{43}$

Aminated QDs have been conjugated, using these homobifunctional coupling agents, to peptides and proteins, ${ }^{72,73}$ lectins,${ }^{74}$ antibodies, ${ }^{75-77}$ receptor ligands ${ }^{78}$ and nucleic acids. ${ }^{79}$

Ruan et al. ${ }^{73}$ employed CdSe/ZnS QDs as fluorescentlabeling probes to target the phosphatidylserine exposed in the plasma membrane, due to drug-induced apoptosis. This bioprocess was monitored by a phospholipid-binding protein named annexin $\mathrm{V}(\mathrm{AV})$. For the conjugation of amino-QDs with $\mathrm{AV}, \mathrm{BS}^{3}$ was used as the coupling reagent. The conjugates were characterized using fluorescence correlation spectroscopy (FCS) and electrophoresis. The QD-AV conjugate was incubated with normal and apoptotic PANC-1 cells and analyzed by fluorescence microscopy and flow cytometry. The results showed that QD-AV conjugates were efficient in apoptosis detection.

In 2019, Zhong et al. ${ }^{80}$ used glutaraldehyde as the coupling agent to conjugate $\mathrm{CdS}$ QDs to anti-Escherichia coli antibodies. The CdS QDs were prepared in an aqueous medium, using mercaptoacetic acid (MAA) as the stabilizing agent and, capped with polyethyleneimine (PEI), to introduce amino groups on their surface. These aminated QDs were then reacted with glutaraldehyde and finally incubated with anti-E. coli antibodies. The final conjugates were used in a sandwich-type electrochemical immunosensor and were able to detect $E$. coli in milk samples.

\subsubsection{Thiol-amine coupling}

To link covalently thiol and amine groups a heterobifunctional-coupling agent, possessing one amine-reactive group and one sulfhydryl-reactive group (Scheme 10), is necessary. The reaction with an amine can be achieved using activated-esters, such as the NHS ester. The reaction with sulfhydryl groups can be carried out with several functional groups, such as maleic anhydride derivatives and compounds containing a disulfide linkage. ${ }^{43,57}$

The amine-reactive end usually is an acylating agent and needs a good leaving group, since the reaction occurs via nucleophilic substitution, forming an amide. The sulfhydryl-reactive end is an alkylating agent that reacts originating either a thioether or a disulfide linkage. Usually, the sulfhydryl-reactive residue is more stable in aqueous media than the amine-reactive group. Thus, these coupling agents are used in a multistep protocol, reacting firstly with the amine-containing QDs, followed by the reaction with the biomolecule thiol groups. One disadvantage of this methodology is that free sulfhydryl groups in biomolecules are rare and they are unstable in the presence of oxygen. ${ }^{43,57}$

Among the most frequently used functional groups in the thiol-reactive end are maleic anhydride derivatives (called maleimides). The sulfhydryl group acts as a nucleophile, attacking the maleimide double bond, with the formation of a thioether. The ideal $\mathrm{pH}$ for this reaction is 6.5-7.5 (Scheme 11a). For the conjugation of QDs coated with amine groups to proteins containing a cysteine residue, the main heterobifunctional coupling agents used are succinimidyl-4-( $N$-maleimidomethyl)cyclohexane1-carboxylate (SMCC, Scheme 11b). ${ }^{43}$

Other thiol-reactive groups used in heterobifunctional coupling agents are compounds containing disulfide bridge $(-\mathrm{S}-\mathrm{S}-)$, which can participate in substitution reactions with sulfhydryl groups. The thiol group attacks

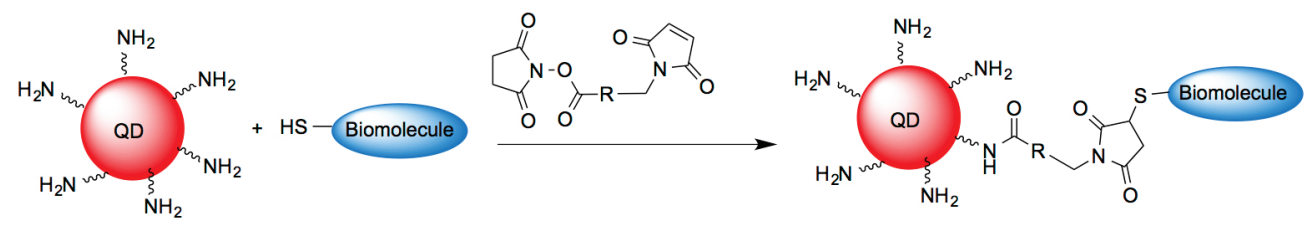

Scheme 10. Schematic representation of QDs conjugation to biomolecules by amine-thiol coupling. 
(a)

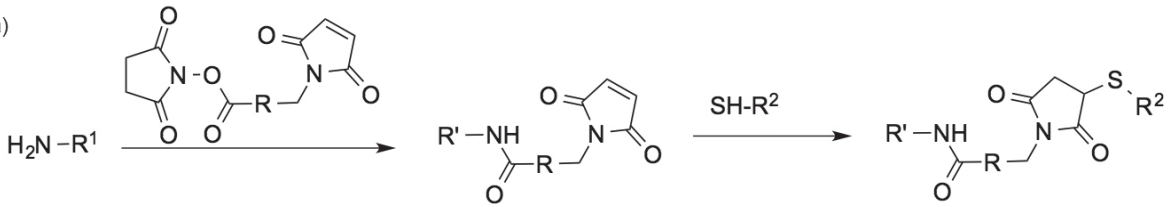

(b)

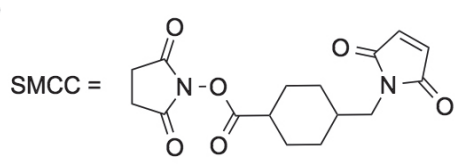

Scheme 11. (a) Reaction steps for the amine-thiol coupling using an heterobifunctional coupling agent containing a NHS ester and a maleimide derivative; (b) chemical structure of the coupling agent SMCC.

the disulfide bond, breaking this original disulfide link and forming a new disulfide bond. A widely used compound is a heterobifunctional reagent containing a pyridyldisulfide residue (Scheme 12a). The main coupling agent used for the conjugation of aminated QDs with proteins, by this approach, is the $\mathrm{N}$-succinimidyl-3-(2-pyridyldithio) propionate (SPDP, Scheme 12b). ${ }^{43}$

This conjugation strategy needs to be carried out in a two-step procedure and, as mentioned before, firstly by the amine-reactive end followed by the addition of the thiol-containing compound. The thiol-reactive intermediate is not stable in an aqueous medium and can also react with sulfhydryl present in the biological medium. ${ }^{43,57}$ Nevertheless, the amine-thiol coupling strategy has been used to covalently link QDs, especially to antibodies ${ }^{41,81}$ and proteins. ${ }^{82,83}$

An example of the use of SPDP for QDs bioconjugation was described by Speranskaya et al. ${ }^{84}$ These authors prepared CdSe QDs in an organic medium, followed by a water compatibilization step, coating the QDs' surface with polymers containing amine groups. Thiolated deoxynivalenol-ovalbumin (DON-OVA) was added to the SPDP coupling agent, forming an amine-reactive intermediate, which was then reacted with QDs- $\mathrm{NH}_{2}$. The conjugates were used in immunoassays and were capable of detecting the mycotoxin deoxynivalenol in wheat and maize.
Recently, Brunetti et al. ${ }^{85}$ conjugated commercial QDs- $\mathrm{NH}_{2}$ to tetra-branched NT4 peptides, which presented potential as cancer theranostics. Firstly, the QDs- $\mathrm{NH}_{2}$ were conjugated with sulfo-SMCC, forming a thiol-reactive QD that was added to the NT4 peptides. The covalent bond was established by the reaction with the thiol group from a terminal cysteine, present in the sequence of the peptides. These conjugates were characterized using nuclear magnetic resonance spectroscopy (NMR), TEM, dynamic light scattering (DLS) analyses and absorbance/emission spectroscopies. Applying flow cytometry analyses, the incubation of QDs-NT4 with human colon adenocarcinoma cell line (HT29) showed specific labeling.

\subsubsection{Alcohol-thiol coupling}

The conjugation between an alcohol and a thiol can be achieved using a heterobifunctional group, containing a hydroxyl-reactive site and a sulfhydrylreactive group. As mention above, reactions with thiol groups can be performed using maleic anhydride derivatives and compounds containing a disulfide bridge. The reaction with hydroxyl-containing molecules or particles can be accomplished using isocyanate groups $(-\mathrm{N}=\mathrm{C}=\mathrm{O})$. Isocyanates react with hydroxyl residues forming a carbamate linkage and can also react with amines to form ureas. These hydroxyl-reactive groups have excellent reactivity, but their stability in water

(a)

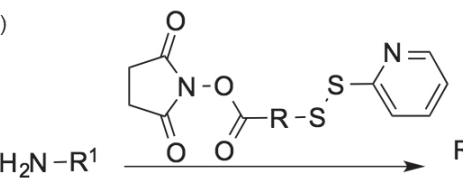<smiles>[R]NC(=O)O[R]</smiles>

(b)

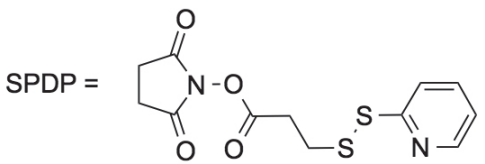

Scheme 12. (a) Reaction steps for the amine-thiol coupling using a heterobifunctional coupling agent containing a NHS ester and a pyridyldisulfide derivative; (b) chemical structure of the coupling agent SPDP. 
is weak. Thus, bioconjugation by this approach needs to be carried out in a two-step procedure. In an initial step, isocyanate reacts with the hydroxyl-containing species, generating a sulfhydryl-reactive intermediate for subsequent conjugation with the thiol-containing species (Scheme 13a). Once more, the thiol-reactive intermediate may react with biological compounds that contain thiol groups. The optimal conditions for this reaction require an alkaline $\mathrm{pH}$ (ca. 8.5). The major heterobifunctional cross-linker used, to associate NPs with biomolecules using this strategy, is the $N$-( $p$-maleimidophenyl) isocyanate (PMPI, Scheme 13b). ${ }^{43,86}$

To the best of our knowledge, only one research group has reported the use of PMPI cross-linker to conjugate hydroxyl-capped QDs with thiolated antibodies (Scheme 14). ${ }^{87,88}$

In 2012, Sukhanova et al. ${ }^{87}$ conjugated CdSe/ZnS QDs, coated with hydroxyl-functionalized PEG polymer, with $\mathrm{IgG}$ antibodies (Abs) from Llama linked to a cysteine residue, using PMPI as the coupling agent. The conjugates were characterized using DLS analyses and flow cytometry. The flow cytometry results showed the specificity of the QDs-Abs to target carcinoembryonic antigen (CEA). The QDs-Abs conjugates were tested as an immunohistochemical diagnostic probe for human biopsy samples and the results showed similar immunolabeling quality to the gold standard protocol. The same research group $^{88}$ in 2014 , following the same approach, linked hydroxyl-coated QDs with Abs from camelid IgG and used them successfully for imaging human appendix and colon carcinoma tissues.

\subsection{Bioorthogonal covalent reactions}

The ultimate goal of any bioconjugation strategy is to occur as a total chemoselective process. Biomolecules and biological systems possess an incredible variety of functional groups and reactive sites, which can cause undesired side reactions. Coupling agents are intended to be specific for a certain reaction, however, most of them also react with other functional groups. For example, maleimide derivatives, described as thiol-reactive cross-linkers, can also react with amines. The NHS ester, used as an amine-reactive coupling agent, reacts as well as with sulfhydryl and hydroxyl groups. To overcome this issue, a reactivity-based bioorthogonal chemistry approach has been developed to build stable covalent bonds for bioconjugation. ${ }^{43,89,90}$

Bioorthogonal reagents should have a reactive-site that only reacts with a specific functional group, without any side-reactions with other functionalities. Ideally, when added to biomolecules or to a complex biological system (in the intracellular medium or serum) these reagents should be stable, not hydrolyze or oxidize, and should react only with the desired functional group. ${ }^{43,89,90}$ Several organic reactions have been classified as bioorthogonal reactions, however, concerning QDs' bioconjugations, $\mathrm{Cu}^{\mathrm{I}}$-catalyzed cycloaddition of azides and terminal alkynes, Diels-Alder cycloadditions involving tetrazine and alkenes and, hydrazine-aldehyde cross-links have been applied.

\subsubsection{Click-chemistry}

A bioorthogonal strategy frequently used for QDs' bioconjugation is the cycloaddition of azides and alkynes.

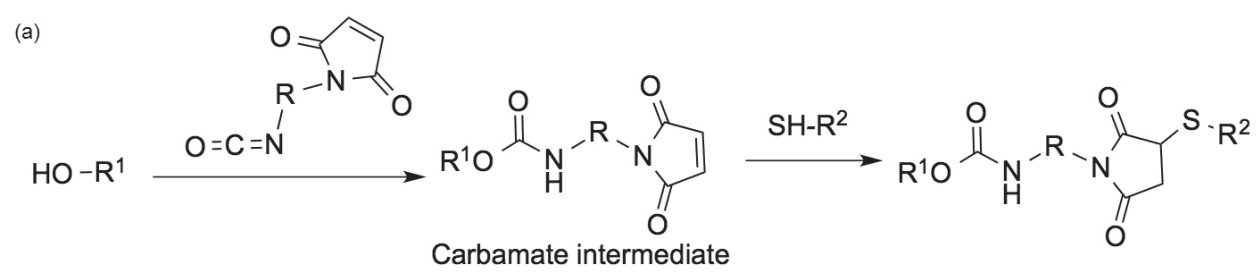<smiles>O=C=Nc1ccc(N2C(=O)C=CC2=O)cc1</smiles>

Scheme 13. (a) Reaction steps for the alcohol-thiol coupling using a heterobifunctional coupling agent containing an isocyanate and a maleimide derivative; (b) chemical structure of the coupling agent PMPI.

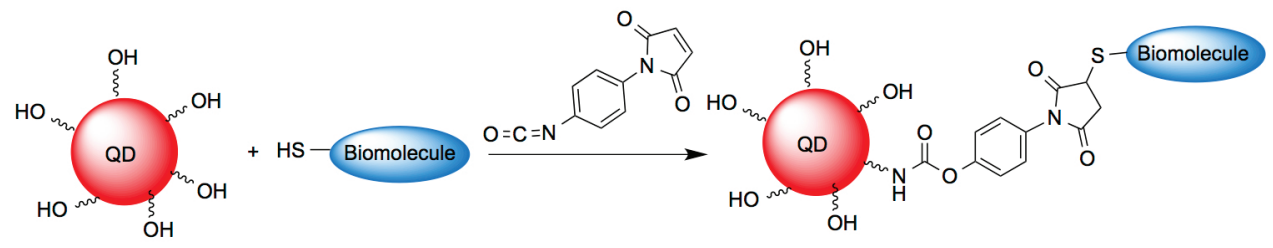

Scheme 14. Schematic representation of QDs conjugation to biomolecules by alcohol-thiol coupling. 
The reaction between an azide and a terminal alkyne, also known as the Huisgen cycloaddition (first developed by Huisgen in 1964), originates a five-membered ring heterocyclic compound containing three nitrogen atoms, called triazole. Originally, this reaction was performed at high temperatures to increase its yield. However, some years later, it was found that this cycloaddition can be catalyzed by $\mathrm{Cu}^{\mathrm{I}}$, originating high yields of the heterocycle ring even at room temperature. For this reason, the $\mathrm{Cu}^{\mathrm{I}}$-catalyzed cycloaddition of azides and terminal alkynes was called a click-chemistry reaction. This concept foresees that a reaction should occur rapidly under ambient conditions in high yield and originate only one product. Besides its high yield and range of applications, this reaction showed to be also bioorthogonal, increasing, even more, its popularity. ${ }^{43,89}$

Azides which react as electrophiles in click reactions, can be easily prepared. Alkyl azides are stable, even in aqueous solution or complex biological media. However, aryl azides are unstable to UV-light or reducing agents. The majority of alkynes are also stable in a biological environment, except the ones containing an electronwithdrawing group (such as a carbonyl group). The introduction of an alkyne group can be simple, such as coupling an activated carboxylic acid with propargylamine, to form an amide bond with a terminal alkyne residue. The triazole ring formed is also stable and not susceptible to hydrolysis or other reactions that could lead to the ring cleavage. ${ }^{43,89}$

The main problem of this $\mathrm{Cu}^{\mathrm{I}}$-catalyzed cycloaddition is that the azide and the alkyne do not react in the absence of copper at room temperature. Also, it has been shown that $\mathrm{Cu}^{\mathrm{I}}$ can induce damage in live cells, thus its cytotoxicity limits the use of this reaction in living systems. To overcome this drawback, Bertozzi and Jewett ${ }^{89}$ developed a Cu-free cycloaddition of azides and alkynes, using cyclooctyne, a ring-strain alkyne. ${ }^{43,89}$

The first study demonstrating QDs' conjugation using the click-chemistry strategy was reported by Binder et al. ${ }^{91}$ in 2007. These authors functionalized QDs either with azides or with alkynes and reacted them with azide or alkyne-supramolecular receptors, respectively, in the presence of $\mathrm{Cu}^{\mathrm{I}}$. Since then, the click-chemistry approach has been growing in QDs' bioconjugation area, especially the copper-free methodologies (Scheme 15). QDs have been coated either with azides or alkynes and conjugated with proteins, ${ }^{92,93}$ viruses,${ }^{94}$ antibodies ${ }^{95,96}$ and other small molecules ${ }^{97,98}$ properly functionalized.

Zhang et al. ${ }^{94}$ in 2012, conjugated QDs to a virus using the click-chemistry strategy. For this, the hydrophobic CdSe/ZnS QDs were coated, by a ligand-exchange methodology, with a polymer containing imidazole and azide groups. Baculovirus (a widely-used vector of gene delivery) was functionalized with dibenzylcyclooctyne (DBCO), by reacting the virus with a cyclooctyl-pegylated polymer. Addition of the QDs- $\mathrm{N}_{3}$ to the alkyne-virus resulted in the QDs-virus conjugation, which was confirmed by fluorescence imaging studies.

In 2016, Ma et al. ${ }^{96}$ prepared lipophilic polymer $\mathrm{CdSe} / \mathrm{ZnS}$ QDs in an organic phase. Some of the oligoethylene glycol (OEG) groups present in the polymer chains had azides at their extremities, enabling their applications in click-chemistry methodologies. These functionalized QDs were mixed with DNA oligomers linked to DBCO, at room temperature. The efficiency of this bioconjugation was confirmed by electrophoresis, labeling QD-DNA conjugates with fluorophores and analyzing them by absorption spectroscopy. The QDs- $\mathrm{N}_{3}$ were also conjugated with DBCO-functionalized IgG and epidermal growth factor receptors (EGFR) antibodies. QDs-IgG and QDs-EGFR conjugates were incubated with A431 melanoma cancer cells, and it was observed that only QDs-EGFR conjugates were bound to the cell membranes, indicating that these conjugates did not present unspecific binding.

Another example is the work of Trapiella-Alfonso et al..$^{29}$ In this study, the authors proposed nanoprobes based on QDs linked to peptides employing bioorthogonal coupling through the azide-alkyne click chemistry. After transferring hydrophobic $\mathrm{ZnCuInSe/ZnS} \mathrm{QDs} \mathrm{to} \mathrm{an} \mathrm{aqueous} \mathrm{medium}$ using a two-step ligand exchange methodology the authors inserted azide functions at the QDs' surface by applying zwitterionic polymers. To enable the reaction with QDs- $\mathrm{N}_{3}$, arginylglycylaspartic acid (RGD) peptides were linked to DBCO and added to the nanocrystals, in the absence of $\mathrm{Cu}^{\mathrm{I}}$ ions. The bioconjugates were characterized using electrophoresis and the labeling of cancer cells, which overexpress $\alpha_{v} \beta_{3}$-integrins, in vitro. The QDs-RGD conjugates were also tested in vivo to study tumor

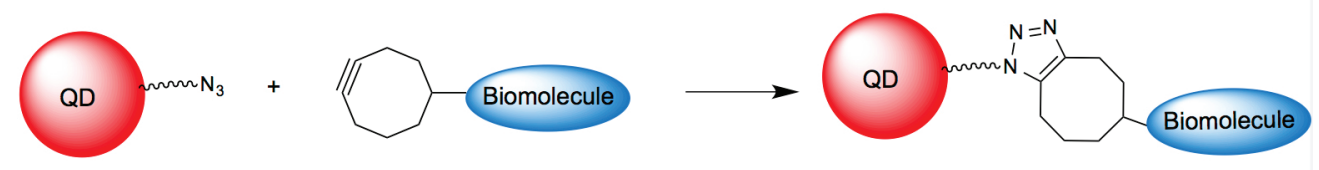

Azide-coated QDs

Cyclooctyne-functionalized biomolecule

Scheme 15. Schematic representation of QDs conjugation to biomolecules by a $\mathrm{Cu}^{\mathrm{I}}$-free cycloaddition of azides and alkynes. 
accumulation in mice, however, the results showed nonspecific labeling, which was attributed to angiogenesis and vascularization mechanisms.

\subsubsection{Hydrazine-aldehyde linking}

Another bioconjugation strategy that is appealing from a bioorthogonal perspective is the reaction between hydrazine derivatives and aldehydes or ketones. Hydrazine derivatives react rapidly and specifically with aldehyde or ketone functional groups, forming a hydrazone bond, which is a type of Schiff base. This reaction is faster with aldehydes than with ketones, however, the hydrazone bonds formed with ketones are more stable than the ones formed with aldehydes. Nevertheless, the hydrazone bond is more stable than the Schiff base formed between aldehydes and amines (section 2.2.2.). ${ }^{43,99}$

The main advantage of this strategy is that biological systems, usually, do not have aldehyde and hydrazine groups. However, biological systems may contain amines that can react with aldehydes. Nevertheless, the Schiff base formed is not stable in an aqueous medium and this linkage can be reversible; the aldehyde will react quickly with the hydrazine present. Thus, the only possibility of cross-linking for this reaction, in a biological medium, would be with aldehydes or ketones generated by cell metabolic cycles, such as reduced sugars. ${ }^{43,99}$

This strategy has been used for QDs' conjugation to biomolecules (Scheme 16) and, studies reported in the literature describe that the nanocrystals have been functionalized with either the aldehyde or the hydrazine group. The hydrazine-aldehyde coupling has been used to conjugate QDs to antibodies, ${ }^{100,101}$ synthetic peptides ${ }^{102}$ and viruses. ${ }^{16}$ This strategy can also be performed applying oxime derivatives (general formula $\mathrm{R}-\mathrm{C}=\mathrm{N}-\mathrm{OH}$ ) instead of hydrazines. ${ }^{25}$

One example of the use of this approach is the study reported by Iyer et al., ${ }^{101}$ in 2011. Applying aromatic aldehyde and aromatic hydrazine derivatives, these authors linked hydrophobic CdSe/ZnS QDs to the anti-EGFR antibody. Firstly, hydrophobic QDs were coated with primary amine-terminated peptides, which further reacted with PEG containing an amine-reactive site in one end of the molecule and an aromatic aldehyde, in the other end. Anti-EGFR antibodies were functionalized with aromatic hydrazines, using a heterobifunctional-coupling agent containing a hydrazine moiety and a NHS ester that was linked to an amine group of the antibody. Mixing the QDs-aldehyde and anti-EGFR-hydrazine ( $\mathrm{pH}$ ca. 6), the biaryl hydrazone bioconjugate was obtained. The conjugation was monitored by absorption spectroscopy, observing the increase in the absorbance at 350 and $390 \mathrm{~nm}$, corresponding to the biaryl hydrazone bond. The QDs-antiEGFR conjugates were used to successfully stain MCF-7 (breast) and U87 cancer cells.

In 2015, Hong et al. ${ }^{16}$ conjugated CdSe QDs to H9N2 virus, a type of avian influenza $\mathrm{A}$ virus that present risk to human health. Commercial CdSe QDs were coated with PEG- $\mathrm{NH}_{2}$ and the modified particles reacted with succinimidyl 4-formylbenzoate (NHS-4FB), obtaining the aldehyde-modified QDs. The virus was functionalized with hydrazine using succinimidyl 6-hydrazinonicotinate acetone hydrazone (NHS-HyNic). This reagent was coupled to amino groups present in the virus by the NHS residue, leaving the hydrazine groups free for further conjugations. The QDs-virus conjugation was accomplished by the reaction between QDs-aldehyde and virus-hydrazine, and the conjugates were characterized using zeta potential measurements, electrophoresis, infrared spectroscopy and TEM microscopy. Fluorescence microscopy analysis showed the effective labeling of the virus by this approach. Furthermore, comparative studies with bioconjugates obtained via the biotin-streptavidin strategy showed that the hydrazine-aldehyde conjugates presented higher labeling efficiency.

\subsubsection{Diels-Alder cycloadditions involving tetrazines and alkenes}

A recent approach of bioorthogonal reactions applied to QDs bioconjugation is the use of Diels-Alder cycloadditions. Diels-Alder [4 +2] cycloadditions are pericyclic reactions between a diene and a dienophile, forming two $\mathrm{C}-\mathrm{C}$ bonds and a six-membered cyclic compound. Normal Diels-Alder reactions are favored by electron-rich dienes and electronpoor alkenes (dienophile), and none catalyst is required. A bioorthogonal strategy that has been developed for bioconjugation is the inverse-electron-demand DielsAlder cycloaddition, involving tetrazines and strained alkenes. In this reaction, tetrazines (electron-poor dienes)

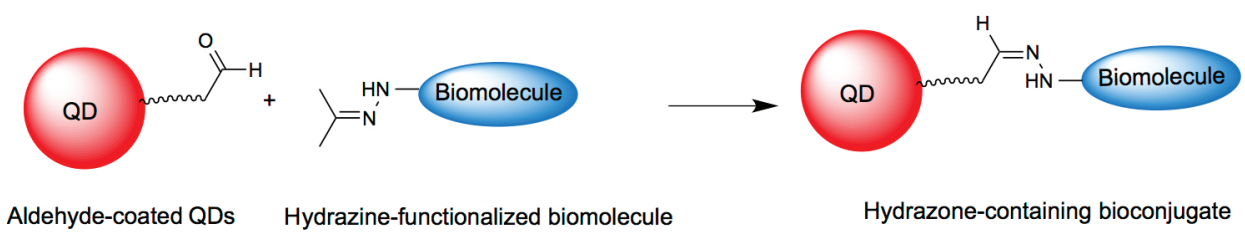

Scheme 16. Schematic representation of QDs conjugation to biomolecules by an aldehyde-hydrazine coupling. 
react with strained alkenes through a Diels-Alder [4 + 2] cycloaddition. The adduct undergoes a further step (a retro Diels-Alder step), releasing nitrogen, and originating the final product, a dihydropyridazine (Scheme 17). This reaction proceeds rapidly in water with a high yield and great selectivity. The reagents and the products formed are also stable in biological media. ${ }^{90,103}$

The main disadvantage of this strategy is the requirement of the attachment of the tetrazine group to the biomolecule, which involves additional coupling steps that could be complex. Another issue is that the functionalization of the QDs and biomolecules, with the needed reactive groups (tetrazine and alkene), can lead to an increase in their hydrophobicity, which could be a drawback. Thus, development of small and hydrophilic reagents that can be used for this bioconjugation strategy is still needed.

Bioconjugation of QDs using this methodology has been developed by Bawendy's research group. ${ }^{28,104}$ In their approach, the nanocrystal was functionalized with the alkene norbornene and the biomolecule was linked to the tetrazine (Scheme 18). These authors prepared the QDs in an organic medium, followed by a ligand exchange procedure with an imidazole-containing polymer covalently linked to norbornene, obtaining the hydrophilic QDs coated with norbornene. The biomolecule was reacted with an aminereactive tetrazine, using $N, N$-dicyclohexylcarbodiimide (DCC) and NHS as coupling agents. QDs bioconjugates were obtained by reacting the QDs-norbornene and the biomolecule-tetrazine for two hours at $37{ }^{\circ} \mathrm{C} .^{28,104}$

In 2010, the same research group functionalized QDs with tetrazine EGF to label EGFRs, which are overexpressed in the membrane of human skin cancer cells. Two approaches were adopted for the cell labeling: using QD-EGF conjugates prepared previously and, performing an in situ conjugation. In the first strategy, the QD-EGF conjugates, obtained by mixing the norbornene-coated QDs with the tetrazine functionalized EGF, were incubated with
A431 human carcinoma cells. In the in situ experiment, the same cell line was incubated with the tetrazine-EGF and then the norbornene-QDs were added (Figure 5). Results obtained by fluorescence microscopy showed that the two approaches labeled cells successfully. ${ }^{104}$

In a second study, in 2015, the same approach was used to conjugate rat anti-mouse monoclonal Abs to QDs. Once coupling the amine-reactive tetrazine to Abs, they were mixed with the norbornene-QDs and, after a purification step, the QD-Ab conjugates were obtained. Since interactions with other biomolecules is a common concern, these authors evaluated the level of the QD-norbornene unspecific interactions in fetal bovine serum (FBS), using FCS measurements. Results showed that the QD-norbornene size remained unaltered and the authors suggested this as an evidence that unspecific adsorption of proteins did not occur. The same study demonstrated that these specific QD-Ab conjugates were successfully applied to label cells. ${ }^{28}$

\section{Methods for Conjugation Evaluation}

To improve the performance and success of the QDs' biological applications it is important to characterize and evaluate the conjugation process efficiency. Among the most popular methods for this purpose we may mention: (i) fluorescence correlation spectroscopy (FCS); (ii) DLS; (iii) electrophoresis; (iv) fluorescence microplate assay (FMA), and (v) zeta potential evaluation, which are described in this section.

\subsection{Fluorescence correlation spectroscopy}

In 1972 Magde, Elson and Webb developed a technique known as fluorescence correlation spectroscopy (FCS) to study the interaction of DNA with drugs using the fluorescence of ethidium bromide. ${ }^{105}$ The FCS technique

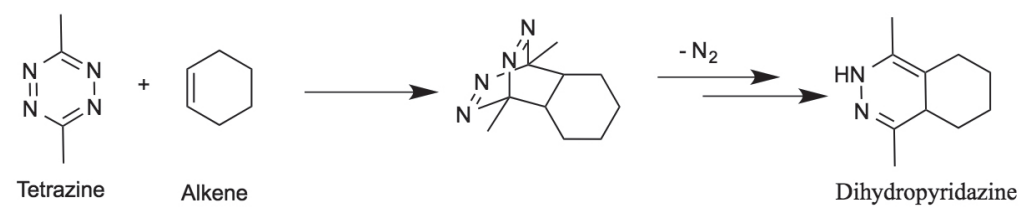

Scheme 17. Diels-Alder cycloaddition reaction between tetrazines and alkenes.

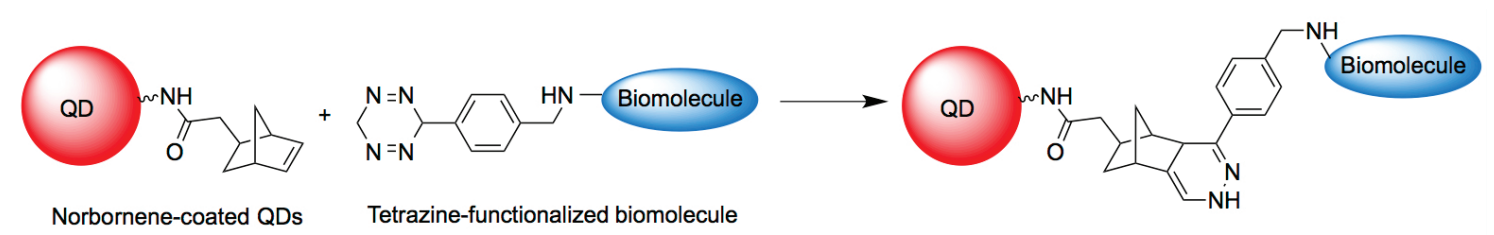

Scheme 18. Schematic representation of QDs conjugation to biomolecules by a Diels-Alder cycloaddition involving tetrazine and alkenes. 
Approach 1
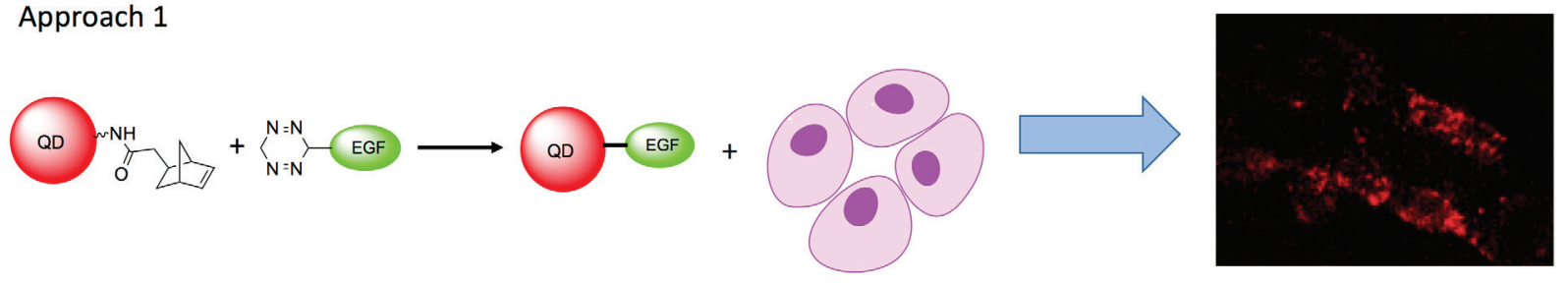

Approach 2
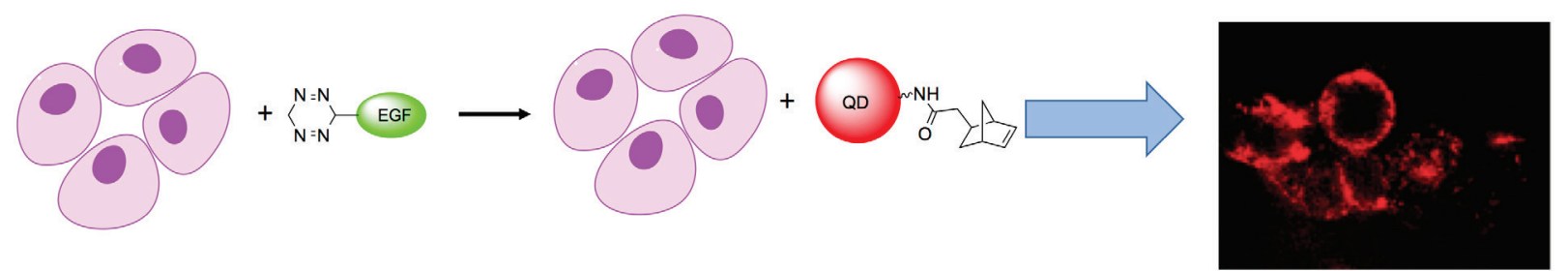

Figure 5. Conjugation of norbornene-coated QDs with the tetrazine functionalized EGF and labeled of skin cancer cells, using two procedures for the Diels-Alder conjugation strategy. Approach 1: preparation of the QDs-EGF conjugates followed by incubation with the cells. Approach 2: incubation of the cells with EGF-tetrazine followed by in situ conjugation with QDs-norbornene (adapted from reference 104 with copyright permission 2010 from ACS Publications).

has been used in a variety of applications such as immunoassays and cell analysis studies, as well as to evaluate the kinetics and thermodynamic parameters of interactions between molecules. ${ }^{106}$

The FCS technique uses a confocal microscope to statistically evaluate the entry and exit of molecules (or particles) in a volume of observation through fluorescence fluctuations. ${ }^{106}$ These fluorescence fluctuations, from the volume of interest, are detected by a photomultiplier in the photon counting regime, generating a real-time electronic signal that is used to construct an autocorrelation curve as a function of time. FCS has been increasingly used as a highly sensitive technique since it works with a nanomolar (nM) sample concentration. FCS provides information related to the size, number of fluorescent molecules or particles, diffusion velocity, as well as interactions between molecules. ${ }^{107,108}$ Thus, FCS has been exploited to quantitatively evaluated the QD-molecules interactions by using the diffusion time and the hydrodynamic radius (R) of bare nanocrystals compared with the conjugates. ${ }^{109}$ The $\mathrm{R}$ can be calculated from equation 1 ,

$\mathrm{R}=\frac{4 \mathrm{k}_{\mathrm{B}} \mathrm{T} \tau_{\mathrm{D}}}{6 \pi \eta \omega_{\mathrm{x}}^{2}}$

where $k_{B}$ is the Boltzmann constant, $T$ is the temperature, $\eta$ is the viscosity of the medium, $\tau_{\mathrm{D}}$ is the diffusion time, and $\omega_{x}$ is the lateral focal volume radius obtained by calibrating the system with a solution of a dye with the diffusion coefficient known, such as rhodamine B. ${ }^{106}$ To show what we should expect from FCS analyses, illustrative autocorrelation curves of bare QDs and conjugates are shown in Figure 6.

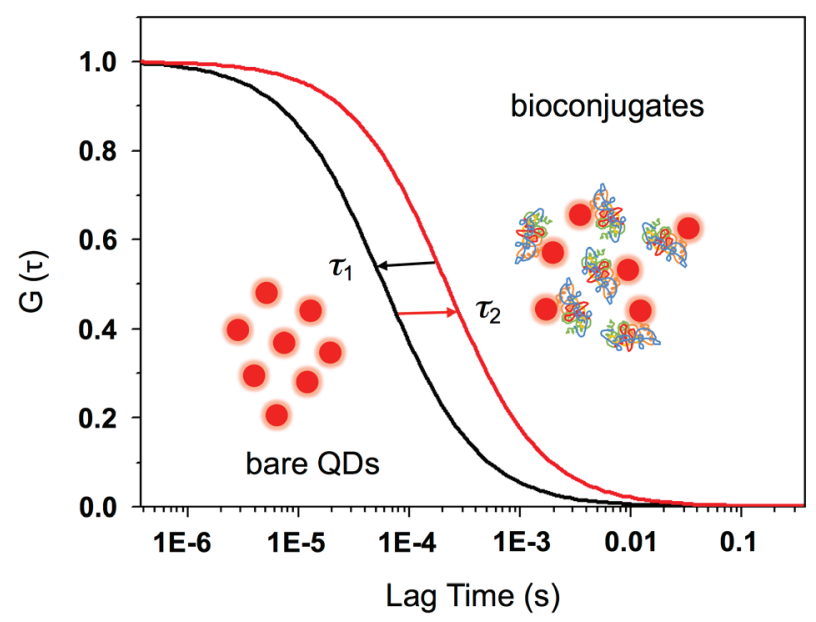

Figure 6. Illustrative autocorrelation curves for bare QDs (in black-faster diffusion) and QDs-bioconjugates (in red-slower diffusion).

Shao et al..${ }^{106}$ using FCS, observed that CdTe QDs were mainly bound by electrostatic attraction to BSA once their QDs:BSA conjugates presented a greater hydrodynamic radius of approximately $7.6 \mathrm{~nm}$ when compared to bare QDs (4.4 nm), having also different diffusion times of about 300 and $171 \mu$ s, respectively. Cabral Filho et al.,22 for example, used FCS to confirm and evaluate the conjugation between CdTe QDs with the anti-A and anti-B Abs (which recognize, respectively, the erythrocyte $A$ and $B$ antigens). The authors obtained a hydrodynamic diameter of about $3.8 \mathrm{~nm}$ for bare QDs whereas the conjugates with anti-A and anti-B exhibited a diameter of about 20.4 
and $18.5 \mathrm{~nm}$, respectively. This evaluation confirmed an efficient conjugation that leads to the labeling of practically all $\mathrm{A}$ and $\mathrm{B}$ erythrocytes by the respective Abs. It is also interesting to mention that anti-A and anti-B antibodies are similar molecules, which correlates with the hydrodynamic diameter obtained by FCS that was almost the same.

FCS real time measurements enables the monitoring of bioconjugation processes / chemical reactions ${ }^{110}$ and local viscosity sensing. ${ }^{111}$ Furthermore, this technique can also differentiate stable conjugates from the ones that may be slightly aggregated, since the aggregates do not show well-fitted autocorrelation curves. ${ }^{110,111}$ Thus, FCS proves to be a valuable and sensitive technique to characterize and study bare QDs and their conjugates.

\subsection{Fluorescence microplate assay}

Fluorescence microplate assay (FMA) is an important method, developed by Carvalho and co-workers ${ }^{112}$ that has the advantage of offering a practical, quantitative and fast analysis of several samples at the same time. According to these authors (as shown in Figure 7) the FMA principle is based on the QDs native fluorescence and the affinity of some biomolecules, such as proteins, for polystyrene microplates. In this method, non-conjugated QDs are removed after rinsing, because they do not have affinity for the microplate and, non-conjugated proteins will be adhered to the microplate but will not emit light in the region of the analyses (400-700 nm). Thus, only proteins bioconjugated to QDs will show significantly detectable fluorescence.
The higher the signal, the greater the efficiency of the conjugation procedure. This process can be described in terms of the relative fluorescence (RF), which correlates the signal of conjugates $\left(\mathrm{FL}_{\text {Tess }}\right)$ with the control ones $\left(\mathrm{FL}_{\text {Controls }}\right.$-an average for QDs and biomolecules alone). A $\mathrm{RF}$ greater than $100 \%$ indicates an efficient conjugation and can be estimated by applying equation $2:{ }^{112}$

$\mathrm{RF}(\%)=\frac{\left(\mathrm{FL}_{\text {Test }}-\mathrm{FL}_{\text {Controls }}\right)}{\mathrm{FL}_{\text {Control }}} \times 100$

The bioconjugation of CdTe QDs with a variety of proteins, such as $\mathrm{Tf}$, and also anti-A and anti-B Abs was evaluated using FMA. ${ }^{22,26,112}$ Cabral Filho et al., ${ }^{22}$ for example, obtained RF signals for anti-A and anti-B conjugates of 3,000 and 2,420\%, respectively. As described before, this efficient QD-blood antibodies conjugation resulted in a specific and effective erythrocyte labeling. In addition, Cabral Filho et al. ${ }^{113}$ developed specific multimodal probes based on CdTe QDs and iron oxide NPs associated with Tf. In their study, the authors tested two Tf concentrations, 88 and $162 \mu \mathrm{g} \mathrm{mL}^{-1}$, obtaining an RF of 1,202 and 7,577\%, respectively. Results indicated that the most concentrated Tf sample promoted the best bioconjugation. The multimodal QD-based nanosystem labeled specifically about $88 \%$ of human cervical carcinoma (HeLa) cells by flow cytometry and it was also an effective negative MRI contrast agent. Therefore, this technique can be also used to find the best biomolecules concentrations and experimental conditions for their association with QDs.

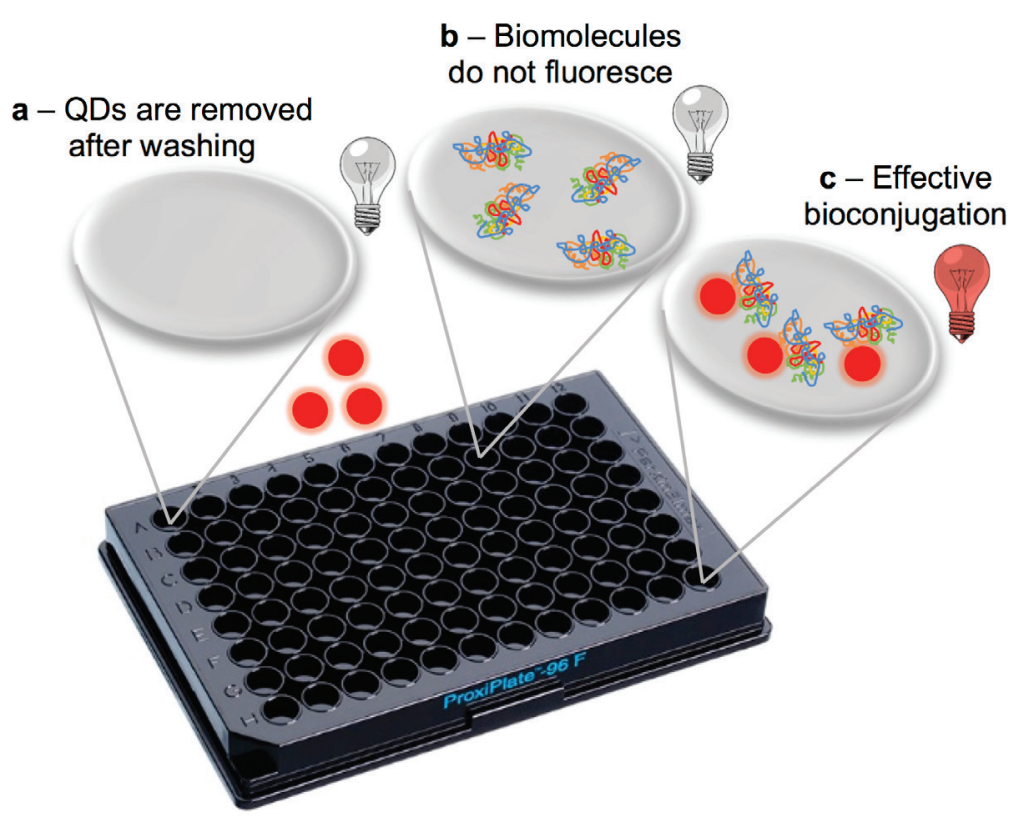

Figure 7. Schematic representation of the fluorescence microplate reader (FMA) method showing from left to right: (a) free QDs are removed by washing and do not present signal; (b) biomolecules have affinity for the microplate but do not fluoresce under the evaluated conditions; and (c) bioconjugates: the better the conjugation the more intense is the detected signal. 


\subsection{Electrophoresis}

Electrophoresis is a technique routinely used in biology for the separation and quantification of electrically charged biomolecules, such as proteins, DNA, RNA, and has also been useful to evaluate QDs' conjugation with biomolecules. ${ }^{110,114,115} \mathrm{Gel}$ electrophoresis analyzes the bioconjugated samples subjected to an electrical voltage, which favors the separation of the systems by differential migration based on their size and charge. By comparing the electrophoretic mobility of the components alone (biomolecules or QDs) in relation to the conjugates, it is possible to evaluate the interaction between QDs and biomolecules. ${ }^{116}$ This technique presents, as principal disadvantages, the analyses of few samples at a time and, in general, it is laborious as well as non-quantitative. ${ }^{115}$

The polyacrylamide gel electrophoresis (PAGE), schematized in Figure 8a, is mostly indicated for conjugation confirmation and/or evaluation studies involving protein-conjugated QDs.22,117 This method is based on the differential migration of the nanocrystals and bioconjugates. Bare QDs migrate faster than the biomolecule conjugates since the latter are larger and heavier. Thus, when QDs are effectively associated with proteins only a single band is observed on the gel. On the other hand, if the conjugation is not complete, the gel will present more than one band referring to conjugates and bare QDs. Chouhan et al., ${ }^{118}$ for example, confirmed a covalent bioconjugation between CdTe QDs and BSA by both native PAGE and sodium dodecyl sulfate (SDS) PAGE. They observed effects of a high negative charge, under native conditions of the conjugates (QDs-BSA) that increased their mobility when compared to the standard BSA. This difference was not observed in SDS-PAGE, because SDS masked the protein charges. Cabral Filho et al. ${ }^{22}$ also confirmed QDs conjugations to anti-A and anti-B Abs by applying PAGE. These authors did not observe any QDs' single band in the conjugated samples, showing a successful conjugation. This result was confirmed by analyzing the bright labeling pattern of erythrocytes by fluorescence microscopy, as can be observed for example in Figure $8 \mathrm{~b}$.

Although PAGE technique offers more uniform pore sizes and higher resolution, most of the conjugated QDs are characterized in agarose gel due to their easier preparation. ${ }^{119}$ Agarose gel electrophoresis has been used in the evaluation of QDs' stability, ${ }^{114}$ in the separation of bare QDs from those conjugated to DNA molecules as well as in the confirmation of the QD-DNA interaction. ${ }^{20,120,121}$ Agarose gel electrophoresis can also be used to analyze conjugation with Abs or their fragments and other biomolecules, such as peptides..$^{21,119}$ Using this technique, Borghei et al. ${ }^{20}$ distinguished complexes based on CdTe QDs and wild or mutated DNA sequences.

In capillary electrophoresis (CE), the sample is injected into one end of the capillary tube and, when submitted to an electrical voltage, it moves through the buffer being detected when reaching the other end. In comparison with traditional electrophoresis, CE is more sensitive, can be semi-quantitative, has the possibility of automatization and can generate greater quality and faster analyses. Generally, changes of intensity and quantity of peaks according to the migration time are recorded by electropherograms. If the analyses present a great number of peaks, it indicates that there are conjugates with a different number of biomolecules associated with each QD, due to differential migration times. Thus, the electropherogram enables the differentiation between QDs and their bioconjugates, because bare QDs present a shorter retention time, whereas conjugates would have longer retention times in the capillary tube. ${ }^{110}$ Wang et al. ${ }^{110}$ for example, monitored, simultaneously, QDs and their assembly and disassembly with glutathione S-Transferase using CE. Datinská et al., ${ }^{122}$

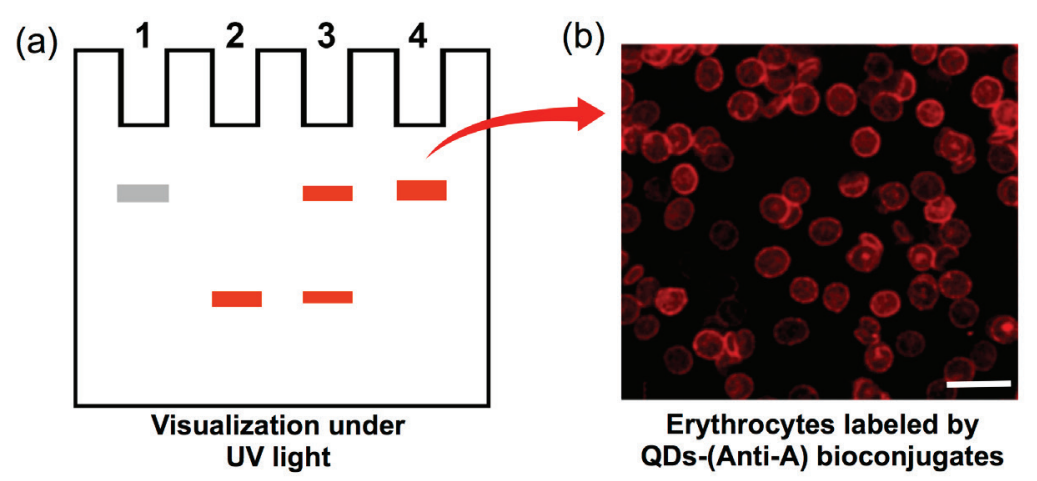

1-biomolecule 2 -bare QDs 3 - ineffective bioconjugation 4 -best bioconjugation

Figure 8. (a) Representation of gel electrophoresis containing only biomolecules (1), bare QDs (2), non-effective conjugation (3) and efficient conjugation (4). (b) Erythrocytes of A blood type labeled with effective QDs-(Anti-A) conjugates under fluorescence microscopy-unpublished image from NanoBio research group. Scale bar: $10 \mu \mathrm{m}$. 
estimated a stoichiometry distribution of approximately one to four molecules of oligonucleotides conjugated to the QDs' surface by this method.

\subsection{Dynamic light scattering and zeta potential}

DLS is a popular technique used to determine the hydrodynamic diameter of particles in suspension at a given temperature and viscosity. In DLS, the sample is inserted in a special cuvette and is illuminated by a monochromatic light source, e.g., HeNe laser. Particles present in the sample are responsible for scattering the incident light. DLS technique analyzes the particle diffusion due to their Brownian motion. ${ }^{67}$ Biomolecules conjugated to QDs can be characterized by assessing differences in DLS hydrodynamic diameter. Although, with a lower sensibility, when compared with FCS, an increase in DLS size for the conjugate is also a good indication that an efficient bioconjugation was carried out.

Another interesting tool to determine the efficiency of a conjugation process is zeta potential evaluation. In aqueous media, charged particles are surrounded by oppositely charged ions present in the solution, i.e., an electrical double layer consisting of the counter-ions is formed around the particles. The Stern layer (inner region) and the diffuse layer (outer region) make up this electrical double layer. Ions strongly attached to the particles are found in the Stern layer whereas ions loosely bound to the particles are in the diffuse layer. A "virtual" border within the diffuse layer, for which the ions move together with the NPs when an electrical voltage is applied, is also referred to as slipping plane (Figure 9a). The electric potential formed at this interface is defined as zeta potential $(\zeta) .{ }^{123}$

To determine the zeta potential an electrical voltage is applied in a special cuvette containing the charged NPs in suspension. The NPs move towards the electrode of opposite polarity at a constant velocity that is proportional to surface charges and consequently the zeta potential. This movement is also monitored by light scattering. Zeta potential also provides information of the colloidal stability of the system. A suspension with good colloidal stability $(\zeta>+20 \mathrm{mV}$ or $<-20 \mathrm{mV})$ should not show aggregation, as a result of mutual electrostatic repulsion between the particles. ${ }^{124}$ In addition, a modification process on QDs' surface (e.g., ligand addition) could be also evaluated using zeta potential analysis. ${ }^{123}$

Ipe et al. ${ }^{125}$ reported the use of DLS and zeta potential techniques to confirm the protein cytochrome $\mathrm{P} 450_{\mathrm{BS} \beta}$ conjugation with $\mathrm{CdS}$ and CdSe QDs. Upon protein conjugation to CdS QDs' surface, for example, increasing in the hydrodynamic diameter (near to three-fold) and modification in the nanoparticles charge from about $\zeta=-28$ to $-5 \mathrm{mV}$ were observed. The authors demonstrated a linear correlation between the quantities of the protein per QD with zeta potential evaluation, that is, higher zeta potential values were observed for larger amounts of attached proteins. Aptamers conjugated to QDs have also been prepared and assessed as a fluorescent nanoprobe for breast cancer cell imaging as well as detection. ${ }^{126}$ In this study, CdSe/ZnS QDs functionalized with maleimide were conjugated to thiol-modified aptamers. The bioconjugate displayed a more negative zeta potential value due to the presence of negatively-charged aptamers on the QDs surface. The aptamer addition was also evidenced by DLS with an increment in the average hydrodynamic size from about 17 to $27 \mathrm{~nm}$ for the QDs and their bioconjugates, respectively. The fluorescent nanoprobes showed to be promising for in vitro diagnostic and bioimaging since differentiated labeling between the breast cancer and normal fibroblast cells were evidenced.

Cabrera et al. ${ }^{36}$ prepared bimodal nanosystems (BNPs) based on QDs conjugated to FF NPs. Firstly, the authors used zeta potential analyses to show the surface modification of the magnetic NPs by APTES, observing a variation from $\zeta=-23$ to $+23 \mathrm{mV}$, as schematized in Figures $9 \mathrm{a}$ and $9 \mathrm{~b}$. After conjugation to QDs, the authors described a negative value of $c a . \zeta=-27 \mathrm{mV}$ as evidence that the positively-charged FF NPs were coated with negatively-charged QDs. Figure 9c shows the optical and magnetic properties of the BNPs. The authors revealed the BNPs as promising MRI and fluorescent nanoprobe promoting efficient cell labeling.

Table 1 shows a compilation of the evaluation bioconjugation methods discussed above. In the case of analyzing the conjugation with surfaces, spectral characterization of solids or electrochemical techniques (such as voltammetry or electrochemical impedance spectroscopy) could, for example, be applied. ${ }^{35}$ It is also worth mentioning that despite the use of these conjugation evaluation techniques, to avoid false positive or false negative results, additional tests (control cells and tissues samples or inhibition and even saturation assays) are necessary. Moreover, depending on the type of the conjugation technique employed, it is also necessary to block the activated functional groups during the bioconjugation process before the application. ${ }^{46,113}$

\section{Conclusion}

Semiconductor QDs have proven to be valuable tools in biology since they were introduced as new a new class of fluorescent probes in 1998. The combination of the 

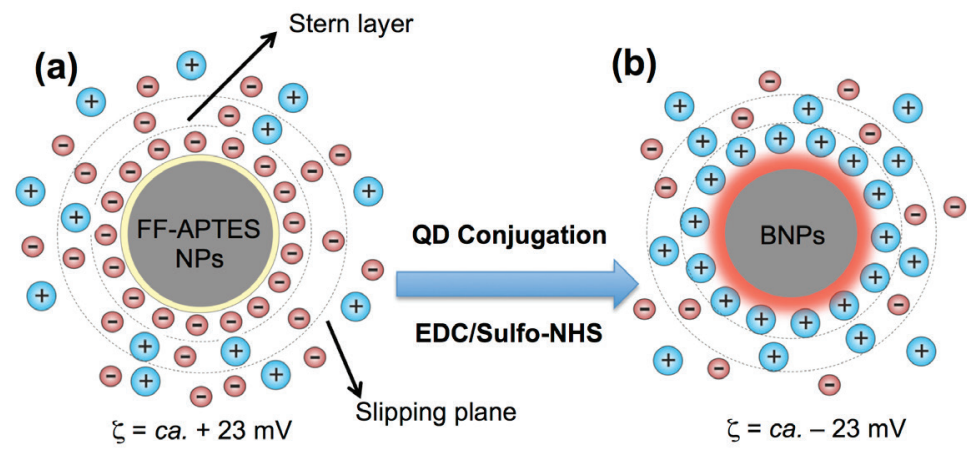

(c) Bimodal system (BNPs)
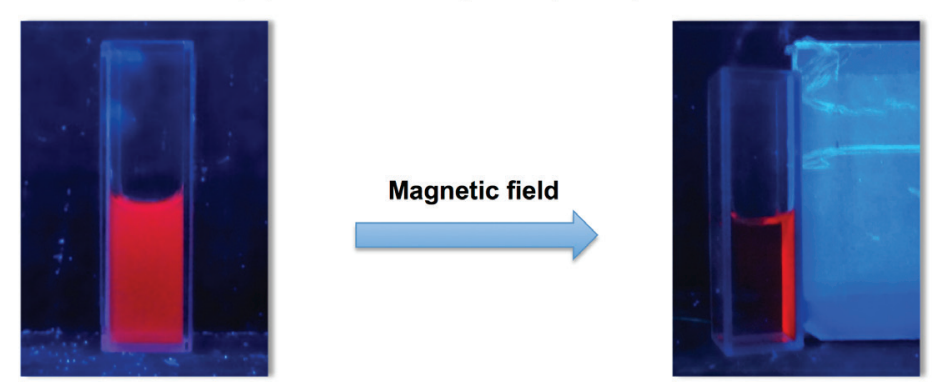

Figure 9. Monitoring the surface modifications in the preparation of BNP particles and optical/magnetic properties. Zeta potential measurements prior (a) and after (b) the conjugation of FF NPs to QDs. (c) Visual evaluation of the fluorescence of BNPs, under UV light, and in the absence of the magnet (left) and in the presence of magnet (right) (unpublished images from the NanoBio research group).

Table 1. Compilation of some methods that can be applied to evaluate the bioconjugation processes

\begin{tabular}{|c|c|c|c|c|}
\hline Method & & Quantitative analyze? & Analyzed parameter & Feature \\
\hline FCS & & yes & hydrodynamic radius and diffusion time & very sensitive ( $\mathrm{nM}$ concentration) and fast \\
\hline FMA & & yes & relative fluorescence signal & facile, multi-samples, and fast \\
\hline \multirow{3}{*}{ Electrophoresis } & PAGE & & & \multirow{2}{*}{ allow for visualization of unconjugated QDs } \\
\hline & agarose & & visual & \\
\hline & capillary & yes & electropherogram & $\begin{array}{l}\text { allow for visualization of unconjugated QDs and } \\
\text { is fast due to automatization }\end{array}$ \\
\hline Zeta potential & & yes & surface charge & sensitive to surface modifications \\
\hline DLS & & yes & hydrodynamic radius & simple method \\
\hline
\end{tabular}

FCS: fluorescence correlation spectroscopy; FMA: fluorescence microplate assay; DLS: dispersion/dynamic light scattering; PAGE: polyacrylamide gel electrophoresis.

QDs' optical properties with the functionalities of the conjugated biomolecules (molecules, coordinating systems, nanoparticles, etc) has enabled their applications in any study conventionally performed with fluorescent organic dyes, such as linear and non-linear microscopy, flow cytometry or even experiments involving förster resonance energy transfer and fluorescence lifetime measurements. These versatile applications have been possible due to the development of several conjugation approaches proposed by adapting known procedures used in organic chemistry. The conjugation strategy needs to be carefully selected and executed, taking into account the biomolecule nature to avoid unspecific labeling or even the loss of the QDs' optical properties. The appropriate coupling agents depend on the functional groups present in the systems of interest (that is, the (bio)molecules, nanoparticles or interfaces) and the QDs' coating surface. Throughout this review, we showed different covalent and non-covalent conjugation strategies currently applied for biological purposes. Additionally, we also discussed that, although the chemical steps of the reactions are well established, the application for determined (bio)systems must be carefully tested. It is necessary to observe that each biomolecule has its own particularities that do not allow the systematization of conjugation methods. Moreover, we presented some of the main methods currently described to evaluate the 
conjugation efficiency, which represent fundamental feedbacks to reach a successful application. Thus, we can conclude that the (bio)conjugation strategies described up to now are versatile and were efficiently applied, but there are still many challenges to be overcome.

\section{Acknowledgments}

The authors acknowledge the Brazilian agencies: Coordenação de Pessoal de Nível Superior (CAPES), Conselho Nacional de Desenvolvimento Científico e Tecnológico (CNPq), Fundação de Amparo a Ciência e a Tecnologia do Estado de Pernambuco (FACEPE) and Instituto Serrapilheira. This work is also linked to the National Institute of Photonics (INCT-INFo), and LARnano/UFPE. The authors also thank Beatriz S. Santos for helping designing the illustrations.

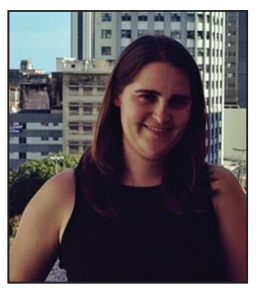

Goreti Pereira is a Professor of the Department of Fundamental Chemistry at the Federal University of Pernambuco (UFPE), since 2016. She has a Bachelor's degree in Physics and Chemistry (2006) and a Doctorate degree in Chemistry (2012) from the University of Minho, Portugal. Her current research focuses on the preparation and application of fluorescent nanocrystals and the synthesis of new amino acids and ligands for magnetic resonance imaging (MRI).

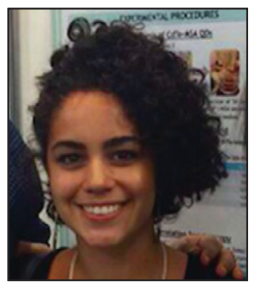

Camila A. P. Monteiro received a Bachelor's degree in Biological Sciences (2016) and is currently pursuing a Master's degree in Biological Sciences at the Federal University of Pernambuco, Brazil. Her research interests focus on the conjugation of quantum dots with biomolecules and their use in biomedical studies.

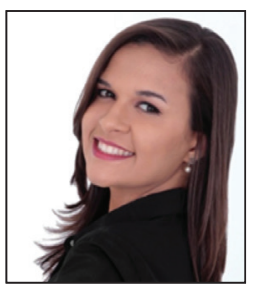

Gabriela M. Albuquerque holds a Bachelor's degree in Chemical Engineering (2018) from the Federal University of Pernambuco, Brazil, where she is currently studying for her Master's degree in Chemistry. She develops new bimodal contrast agents for optical imaging and MRI, based on quantum dots.

Maria I. A. Pereira has a Bachelor's degree in Biomedicine (2014) and a Master's degree in Biological

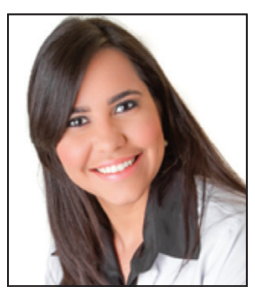

Sciences (2016) from the Federal University of Pernambuco, Brazil, where she is currently a graduate student in Biological Sciences. Her research interest focuses on the development of new multimodal contrast agents for optical imaging and MRI, based on quantum dots.

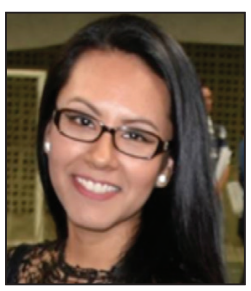

Mariana P. Cabrera received her Bachelor's degree in Bromatology (2007) from the Universidad Nacional de Salta, Argentina. She earned a Doctorate degree in Biological Sciences in 2013 from the Federal University of Pernambuco, Brazil. Her research interests focus on the development and application of magnetic nanoparticles and quantum dots.

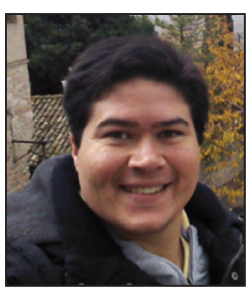

Paulo E. Cabral Filho is a Professor at the Biophysics and Radiobiology Department of the Federal University of Pernambuco since 2017. He received a Bachelor's degree in Biomedicine (2011), and a Master's (2011) and Doctorate (2016) degrees in Biological Sciences from the same University. His research focuses on the preparation and bioconjugation of quantum dots for biomedical applications.

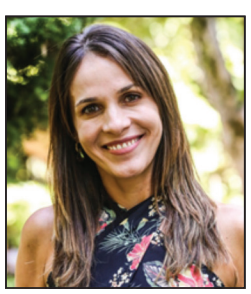

Giovannia A. L. Pereira is a Professor at the Department of Fundamental Chemistry of the Federal University of Pernambuco, Brazil. Dr Pereira completed her graduation in Chemistry at the same University, in 2001, and obtained her Doctorate degree in Biochemistry at the University of Coimbra, Portugal, in 2008. Her research interests are centered on the preparation of hydrophilic inorganic nanocrystals and the development of new multimodal contrast agents for optical imaging and MRI, based on quantum dots.

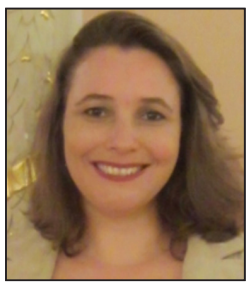

Adriana Fontes is a professor at the Biophysics and Radiobiology Department of the Federal University of Pernambuco since 2006. Her research breakthroughs include the synthesis and application of quantum dots and the use of optical tweezers in 
biomedical sciences. She was awarded the L'Oréal-Brazil for Women in Science Grant in 2008. Dr Fontes received her Bachelor's degree in Physics, in 1996, from the State University of Campinas and her PhD in Science from the same University, in 2004.

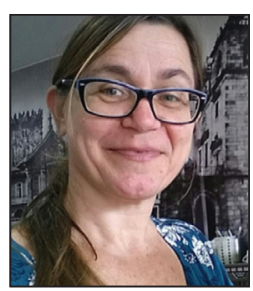

Beate S. Santos is a Professor at the Pharmaceutical Sciences Department of the Federal University of Pernambuco since 2002. Her research interests focus on inorganic colloidal systems, quantum dots and metallic nanoparticles, diagnosis based on fluorescence, the application of fluorescent systems to photodynamic therapy and the study of semisolid formulations for therapeutic applications. Dr Santos has also studied qualitatively and quantitatively the identification of illicit drugs, in collaboration with the Pernambuco State Institute of Criminology. Dr Santos received her Bachelor's degree in Chemistry (1988), MSc (1996) and PhD (2002) degrees in Chemistry all from the Federal University of Pernambuco.

\section{References}

1. Wegner, K. D.; Hildebrandt, N.; Chem. Soc. Rev. 2015, 44, 4792.

2. Pereira, M. G. C.; Leite, E. S.; Pereira, G. A. L.; Fontes, A.; Santos, B. S. In Nanocolloids - A Meeting Point for Scientists and Technologists, $1^{\text {st }}$ ed.; Sanchez-Dominguez, M.; RodriguezAbreu, C., eds.; Elsevier: Amsterdam, 2016, ch. 4, p. 131.

3. Ekimov, A. I.; Onushchenko, A. A.; JETP Lett. 1981, 34, 345.

4. Ekimov, A. I.; Efros, A. L.; Onushchenko, A. A.; Solid State Commun. 1985, 56, 921.

5. Brus, L. E.; J. Chem. Phys. 1984, 80, 6.

6. Bruchez, M.; Moronne, M.; Gin, P.; Weiss, S.; Alivisatos, A. P.; Science 1998, 281, 2013.

7. Chan, W. C. W.; Nie, S.; Science 1998, 281, 2016.

8. Liu, B.; Jiang, B.; Zheng, Z.; Liu, T.; J. Lumin. 2019, $209,61$.

9. Sousa, J. C. L.; Vivas, M. G.; Ferrari, J. L.; Schiavon, M. A.; J. Braz. Chem. Soc. 2018, 29, 2496.

10. Bai, X.; Purcell-Milton, F.; Gun'ko, Y. K.; Nanomaterials 2019 , 9,85 .

11. Jiang, T.; Song, J.; Wang, H.; Ye, X.; Wang, H.; Zhang, W.; Yang, M.; Xia, R.; Zhu, L.; Xu, X.; J. Mater. Chem. B 2015, 3, 2402.

12. Deng, D.; Qu, L.; Achilefu, S.; Gu, Y.; Chem. Commun. 2013, 49, 9494.

13. Fontes, A.; de Lira, R. B.; Seabra, M. A. B. L.; da Silva, T. G.; Neto, A. G. C.; Santos, B. S. In Biomedical Engineering Technical Applications in Medicine; Hudak, R., ed.; InTech, 2012.
14. Liu, X.; Tian, J.; Dai, J.; Wang, X.; Phys. E 2014, 57, 56.

15. Wenger, W. N.; Bates, F. S.; Aydil, E. S.; Langmuir 2017, 33, 8239.

16. Hong, Z.-Y.; Lv, C.; Liu, A.-A.; Liu, S.-L.; Sun, E.-Z.; Zhang, Z.-L.; Lei, A.-W.; Pang, D.-W.; ACS Nano 2015, 9, 11750.

17. Alivisatos, A. P.; Gu, W.; Larabell, C.; Annu. Rev. Biomed. Eng. 2005, 7, 55.

18. Medintz, I. L.; Uyeda, H. T.; Goldman, E. R.; Mattoussi, H.; Nat. Mater. 2005, 4, 435.

19. Sukhanova, A.; Nabiev, I.; Expert Opin. Med. Diagn. 2008, 2 , 429.

20. Borghei, Y.-S.; Hosseini, M.; Ganjali, M. R.; Hosseinkhani, S.; J. Pharm. Biomed. Anal. 2018, 152, 81.

21. Mahmoud, W.; Rousserie, G.; Reveil, B.; Tabary, T.; Millot, J.-M.; Artemyev, M.; Oleinikov, V. A.; Cohen, J. H. M.; Nabiev, I.; Sukhanova, A.; Anal. Biochem. 2011, 416, 180.

22. Cabral Filho, P. E.; Pereira, M. I. A.; Fernandes, H. P.; de Thomaz, A. A.; Cesar, C. L.; Santos, B. S.; Barjas-Castro, M. L.; Fontes, A.; Int. J. Nanomed. 2015, 10, 4393.

23. Cunha, C. R. A.; Andrade, C. G.; Pereira, M. I. A.; Cabral Filho, P. E.; Carvalho, L. B.; Coelho, L. C. B. B.; Santos, B. S.; Fontes, A.; Correia, M. T. S.; J. Photochem. Photobiol., B 2018, 178, 85.

24. Cunha, C. R. A.; Oliveira, A. D. P. R.; Firmino, T. V. C.; Tenório, D. P. L. A.; Pereira, G.; Carvalho, L. B.; Santos, B. S.; Correia, M. T. S.; Fontes, A.; Biochim. Biophys. Acta, Gen. Subj. 2018, 1862, 427.

25. Palomo, V.; Cistrone, P. A.; Zhan, N.; Palui, G.; Mattoussi, H.; Dawson, P. E.; Bioconjugate Chem. 2018, 29, 3144.

26. Cabral Filho, P. E.; Cardoso, A. L. C.; Pereira, M. I. A.; Ramos, A. P. M.; Hallwass, F.; Castro, M. M. C. A.; Geraldes, C. F. G. C.; Santos, B. S.; Pedroso de Lima, M. C.; Pereira, G. A. L.; Fontes, A.; Biochim. Biophys. Acta, Gen. Subj. 2016, 1860, 28.

27. Vieira, C. S.; Almeida, D. B.; de Thomaz, A. A.; Menna-Barreto, R. F. S.; dos Santos-Mallet, J. R.; Cesar, C. L.; Gomes, S. A. O.; Feder, D.; Mem. Inst. Oswaldo Cruz 2011, 106, 158.

28. Han, H.-S.; Niemeyer, E.; Huang, Y.; Kamoun, W. S.; Martin, J. D.; Bhaumik, J.; Chen, Y.; Roberge, S.; Cui, J.; Martin, M. R.; Fukumura, D.; Jain, R. K.; Bawendi, M. G.; Duda, D. G., Proc. Natl. Acad. Sci. U. S. A.2015, 112, 1350.

29. Trapiella-Alfonso, L.; Pons, T.; Lequeux, N.; Leleu, L.; Grimaldi, J.; Tasso, M.; Oujagir, E.; Seguin, J.; d'Orlyé, F.; Girard, C.; Doan, B.-T.; Varenne, A.; ACS Appl. Mater. Interfaces 2018, 10, 17107.

30. Kulkarni, N. S.; Guererro, Y.; Gupta, N.; Muth, A.; Gupta, V.; J. Drug Delivery Sci. Technol. 2019, 49, 352.

31. Tang, M.; Pi, J.; Long, Y.; Huang, N.; Cheng, Y.; Zheng, H.; Spectrochim. Acta, Part A 2018, 201, 82.

32. Yu, F.; Xiong, Y.-M.; Yu, S.-C.; He, L.-L.; Niu, S.-S.; Wu, Y.-M.; Liu, J.; Qu, L.-B.; Liu, L.-E.; Wu, Y.-J.; Int. J. Nanomed. 2018, 13,429 . 
33. Algar, W. R.; Tavares, A. J.; Krull, U. J.; Anal. Chim. Acta 2010, $673,1$.

34. Vannoy, C. H.; Tavares, A. J.; Noor, M. O.; Uddayasankar, U.; Krull, U. J.; Sensors 2011, 11, 9732.

35. Li, C.-c.; Hu, J.; Lu, M.; Zhang, C.-y.; Biosens. Bioelectron. 2018, $122,51$.

36. Cabrera, M. P.; Cabral Filho, P. E.; Silva, C. M. C. M.; Oliveira, R. M.; Geraldes, C. F. G. C.; Castro, M. M. C. A.; Costa, B. F. O.; Henriques, M. S. C.; Paixão, J. A.; Carvalho Jr., L. B.; Santos, B. S.; Hallwass, F.; Fontes, A.; Pereira, G. A. L.; Nanotechnology 2017, 28, 285704.

37. McAdams, S. G.; Lewis, D. J.; McNaughter, P. D.; Lewis, E. A.; Haigh, S. J.; O’Brien, P.; Tuna, F.; Chem. Commun. 2017, $53,10500$.

38. Du, Y.; Rajamanickam, K.; Stumpf, T. R.; Qin, Y.; McCulloch, H.; Yang, X.; Zhang, J.; Tsai, E.; Cao, X.; J. Inorg. Organomet. Polym. Mater. 2018, 28, 711.

39. Samia, A. C. S.; Dayal, S.; Burda, C.; Photochem. Photobiol. 2006, 82, 617.

40. Viana, O.; Ribeiro, M.; Rodas, A.; Rebouças, J.; Fontes, A.; Santos, B.; Molecules 2015, $20,8893$.

41. Jennings, T. L.; Becker-Catania, S. G.; Triulzi, R. C.; Tao, G.; Scott, B.; Sapsford, K. E.; Spindel, S.; Oh, E.; Jain, V.; Delehanty, J. B.; Prasuhn, D. E.; Boeneman, K.; Algar, W. R.; Medintz, I. L.; ACS Nano 2011, 5, 5579.

42. Foubert, A.; Beloglazova, N. V.; Rajkovic, A.; Sas, B.; Madder, A.; Goryacheva, I. Y.; de Saeger, S.; TrAC, Trends Anal. Chem. 2016, 83, 31 .

43. Hermanson, G. T.; Bioconjugate Techniques; Academic press: London, 2013.

44. Xiao, Q.; Huang, S.; Ma, J.; Su, W.; Li, P.; Cui, J.; Liu, Y.; J. Photochem. Photobiol., A 2012, 249, 53.

45. Miao, L.; Han, J.; Zhang, H.; Zhao, L.; Si, C.; Zhang, X.; Hou, C.; Luo, Q.; Xu, J.; Liu, J.; ACS Nano 2014, 8, 3743.

46. Andrade, C. G.; Cabral Filho, P. E.; Tenório, D. P. L.; Santos, B. S.; Beltrão, E. I. C.; Fontes, A.; Carvalho Jr., L. B.; Int. J. Nanomed. 2013, 8, 4623.

47. Coulon, J.; Thouvenin, I.; Aldeek, F.; Balan, L.; Schneider, R.; J. Fluoresc. 2010, 20, 591.

48. Zhao, J.; Qiu, X.; Wang, Z.; Pan, J.; Chen, J.; Han, J.; OncoTargets Ther. 2013, 6, 303.

49. Sapsford, K.; Pons, T.; Medintz, I.; Mattoussi, H.; Sensors 2006, 6,925

50. Aldeek, F.; Safi, M.; Zhan, N.; Palui, G.; Mattoussi, H.; ACS Nano 2013, 7, 10197.

51. Zhan, N.; Palui, G.; Mattoussi, H.; Nat. Protoc. 2015, 10, 859.

52. Tsuboi, S.; Sasaki, A.; Sakata, T.; Yasuda, H.; Jin, T.; Chem. Commun. 2017, 53, 9450.

53. Guo, Y.; Sakonsinsiri, C.; Nehlmeier, I.; Fascione, M. A.; Zhang, H.; Wang, W.; Pöhlmann, W.; Turnbull, W. B.; Zhou, D.; Angew. Chem., Int. Ed. 2016, 55, 4738.
54. Tomczak, N.; Liu, R.; Vancso, J. G.; Nanoscale 2013, 5, 12018.

55. Safi, M.; Domitrovic, T.; Kapur, A.; Zhan, N.; Aldeek, F.; Johnson, J. E.; Mattoussi, H.; Bioconjugate Chem. 2017, 28, 64.

56. Diamandis, E. P.; Christopoulos, T. K.; Clin. Chem. 1991, 37, 625.

57. Zhang, F.; Lees, E.; Amin, F.; Rivera_Gil, P.; Yang, F.; Mulvaney, P.; Parak, W. J.; Small 2011, 7, 3113.

58. Lesch, H. P.; Kaikkonen, M. U.; Pikkarainen, J. T.; YläHerttuala, S.; Expert Opin. Drug Delivery 2010, 7, 551.

59. Tang, J.; Huang, N.; Zhang, X.; Zhou, T.; Tan, Y.; Pi, J.; Pi, L.; Cheng, S.; Zheng, H.; Cheng, Y.; Int. J. Nanomed. 2017, 12, 3899.

60. Theodorou, I. G.; Jawad, Z. A. R.; Qin, H.; Aboagye, E. O.; Porter, A. E.; Ryan, M. P.; Xie, F.; Nanoscale 2016, 8, 12869.

61. Dogan, Ü.; Kasap, E.; Cetin, D.; Suludere, Z.; Boyaci, I. H.; Türkyılmaz, C.; Ertas, N.; Tamer, U.; Sens. Actuators, B 2016, $233,369$.

62. Rousserie, G.; Grinevich, R.; Brazhnik, K.; Even-Desrumeaux, K.; Reveil, B.; Tabary, T.; Chames, P.; Baty, D.; Cohen, J. H. M.; Nabiev, I.; Sukhanova, A.; Anal. Biochem. 2015, 478, 26.

63. Wang, Z.-y.; Wang, L.-j.; Zhang, Q.; Tang, B.; Zhang, C.-y.; Chem. Sci. 2018, 9, 1330.

64. Hu, J.; Liu, M.-h.; Zhang, C.-y.; Chem. Sci. 2018, 9, 4258.

65. Ghimire, S.; Sivadas, A.; Yuyama, K.-i.; Takano, Y.; Francis, R.; Biju, V.; Nanoscale 2018, 10, 13368.

66. Benoiton, N. L.; Chemistry of Peptide Synthesis; Taylor \& Francis Group: Boca Raton, 2006.

67. Lim, J.; Yeap, S. P.; Che, H. X.; Low, S. C.; Nanoscale Res. Lett. 2013, 8, 381.

68. Ren, X.; Xue, Q.; Wen, L.; Li, X.; Wang, H.; Anal. Chim. Acta 2019, 1053, 114.

69. Arumugam, N.; Kim, J.; Mater. Sci. Eng., C 2018, 92, 720.

70. Kalangi, S. K.; Swarnakar, N. K.; Sathyavathi, R.; Narayana Rao, D.; Jain, S.; Reddanna, P.; Oxid. Med. Cell. Longevity 2018, 2018, 8 .

71. Zhang, F.; Xu, L.; Zhao, Q.; Sun, Y.; Wang, X.; Ma, P.; Song, D.; Sens. Actuators, B 2018, 256, 1069.

72. Sun, M.; Yang, L.; Jose, P.; Wang, L.; Zweit, J.; J. Mater. Chem. B 2013, 1, 6137.

73. Ruan, L.; Ge, M.; Huang, X.; Ren, J.; Langmuir 2018, 34, 10040.

74. Wang, J.-H.; Li, Y.-Q.; Zhang, H.-L.; Wang, H.-Q.; Lin, S.; Chen, J.; Zhao, Y.-D.; Luo, Q.-M.; Colloids Surf., A 2010, 364, 82.

75. Veeranarayanan, S.; Poulose, A. C.; Mohamed, M. S.; Nagaoka, Y.; Iwai, S.; Nakagame, Y.; Kashiwada, S.; Yoshida, Y.; Maekawa, T.; Kumar, D. S.; Int. J. Nanomed. 2012, 7, 3769.

76. Kim, C.; Hoffmann, G.; Searson, P. C.; ACS Sens. 2017, $2,766$.

77. Chen, Z.; Lu, M.; Anal. Chim. Acta 2017, 979, 24. 
78. Pati, M. L.; Fanizza, E.; Hager, S.; Groza, D.; Heffeter, P.; Laurenza, A. G.; Laquintana, V.; Curri, M. L.; Depalo, N.; Abate, C.; Denora, N.; Mol. Pharmaceutics 2018, 15, 458.

79. Raichlin, S.; Sharon, E.; Freeman, R.; Tzfati, Y.; Willner, I.; Biosens. Bioelectron. 2011, 26, 4681.

80. Zhong, M.; Yang, L.; Yang, H.; Cheng, C.; Deng, W.; Tan, Y.; Xie, Q.; Yao, S.; Biosens. Bioelectron. 2019, 126, 493.

81. Vazquez, A. L. Q.; Torchynska, T. V.; Casas Espinola, J. L.; Jaramillo Gómez, J. A.; Douda, J.; J. Lumin. 2013, 143, 38.

82. Lei, Y.; Tang, H.; Yao, L.; Yu, R.; Feng, M.; Zou, B.; Bioconjugate Chem. 2008, 19, 421.

83. Gao, J.; Chen, K.; Miao, Z.; Ren, G.; Chen, X.; Gambhir, S. S.; Cheng, Z.; Biomaterials 2011, 32, 2141.

84. Speranskaya, E. S.; Beloglazova, N. V.; Lenain, P.; de Saeger, S.; Wang, Z.; Zhang, S.; Hens, Z.; Knopp, D.; Niessner, R.; Potapkin, D. V.; Goryacheva, I. Y.; Biosens. Bioelectron. 2014, 53, 225.

85. Brunetti, J.; Riolo, G.; Gentile, M.; Bernini, A.; Paccagnini, E.; Falciani, C.; Lozzi, L.; Scali, S.; Depau, L.; Pini, A.; Lupetti, P.; Bracci, L.; J. Nanobiotechnol. 2018, 16, 21.

86. Annunziato, M. E.; Patel, U. S.; Ranade, M.; Palumbo, P.; Bioconjugate Chem. 1993, 4, 212.

87. Sukhanova, A.; Even-Desrumeaux, K.; Kisserli, A.; Tabary, T.; Reveil, B.; Millot, J.-M.; Chames, P.; Baty, D.; Artemyev, M.; Oleinikov, V.; Pluot, M.; Cohen, J. H. M.; Nabiev, I.; Nanomedicine 2012, 8, 516.

88. Hafian, H.; Sukhanova, A.; Turini, M.; Chames, P.; Baty, D.; Pluot, M.; Cohen, J. H. M.; Nabiev, I.; Millot, J.-M.; Nanomedicine 2014, 10, 1701.

89. Jewett, J. C.; Bertozzi, C. R.; Chem. Soc. Rev. 2010, 39, 1272. 90. Devaraj, N. K.; Weissleder, R.; Acc. Chem. Res. 2011, 44, 816.

91. Binder, W. H.; Sachsenhofer, R.; Straif, C. J.; Zirbs, R.; J. Mater. Chem. 2007, 17, 2125.

92. Schieber, C.; Bestetti, A.; Lim, J. P.; Ryan, A. D.; Nguyen, T.L.; Eldridge, R.; White, A. R.; Gleeson, P. A.; Donnelly, P. S.; Williams, S. J.; Mulvaney, P.; Angew. Chem., Int. Ed. 2012, 51, 10523.

93. Mann, V. R.; Powers, A. S.; Tilley, D. C.; Sack, J. T.; Cohen, B. E.; ACS Nano 2018, 12, 4469.

94. Zhang, P.; Liu, S.; Gao, D.; Hu, D.; Gong, P.; Sheng, Z.; Deng, J.; Ma, Y.; Cai, L.; J. Am. Chem. Soc. 2012, 134, 8388.

95. Kotagiri, N.; Li, Z.; Xu, X.; Mondal, S.; Nehorai, A.; Achilefu, S.; Bioconjugate Chem. 2014, 25, 1272.

96. Ma, L.; Tu, C.; Le, P.; Chitoor, S.; Lim, S. J.; Zahid, M. U.; Teng, K. W.; Ge, P.; Selvin, P. R.; Smith, A. M.; J. Am. Chem. Soc. 2016, 138, 3382.

97. Bernardin, A.; Cazet, A.; Guyon, L.; Delannoy, P.; Vinet, F.; Bonnaffé, D.; Texier, I.; Bioconjugate Chem. 2010, 21, 583.

98. Nxele, S. R.; Nyokong, T.; Electrochim. Acta 2016, 194, 26.

99. Blanco-Canosa, J. B.; Wu, M.; Susumu, K.; Petryayeva, E.; Jennings, T. L.; Dawson, P. E.; Algar, W. R.; Medintz, I. L.; Coord. Chem. Rev. 2014, 263-264, 101.
100. Pereira, M.; Lai, E. P. C.; J. Nanobiotechnol. 2008, 6, 10.

101. Iyer, G.; Pinaud, F.; Xu, J.; Ebenstein, Y.; Li, J.; Chang, J.; Dahan, M.; Weiss, S.; Bioconjugate Chem. 2011, 22, 1006.

102. Blanco-Canosa, J. B.; Medintz, I. L.; Farrell, D.; Mattoussi, H.; Dawson, P. E.; J. Am. Chem. Soc. 2010, 132, 10027.

103. Oliveira, B. L.; Guo, Z.; Bernardes, G. J. L.; Chem. Soc. Rev. 2017, 46, 4895.

104. Han, H.-S.; Devaraj, N. K.; Lee, J.; Hilderbrand, S. A.; Weissleder, R.; Bawendi, M. G.; J. Am. Chem. Soc. 2010, 132, 7838.

105. Magde, D.; Elson, E.; Webb, W. W.; Phys. Rev. Lett. 1972, 29 , 705.

106. Shao, L.; Dong, C.; Sang, F.; Qian, H.; Ren, J.; J. Fluoresc. 2009, 19, 151.

107. Krichevsky, O.; Bonnet, G.; Rep. Prog. Phys. 2002, 65, 251.

108. Heuff, R. F.; Swift, J. L.; Cramb, D. T.; Phys. Chem. Chem. Phys. 2007, 9, 1870.

109. de Thomaz, A. A.; Almeida, D. B.; Pelegati, V. B.; Carvalho, H. F.; Cesar, C. L.; J. Phys. Chem. B 2015, 119, 4294.

110. Wang, J.; Huang, X.; Zan, F.; Guo, C.-g.; Cao, C.; Ren, J.; Electrophoresis 2012, 33, 1987.

111. de Thomaz, A. A.; Almeida, D. B.; Cesar, C. L. In Quantum Dots: Applications in Biology; Fontes, A.; Santos, B. S., eds.; Springer New York: New York, 2014, p. 85-91.

112. Carvalho, K. H. G.; Brasil Jr., A. G.; Cabral Filho, P. E.; Tenorio, D. P. L. A.; de Siqueira, A. C. A.; Leite, E. S.; Fontes, A.; Santos, B. S.; J. Nanosci. Nanotechnol. 2014, 14, 3320.

113. Cabral Filho, P. E.; Cabrera, M. P.; Cardoso, A. L. C.; Santana, O. A.; Geraldes, C. F. G. C.; Santos, B. S.; Pedroso de Lima, M. C.; Pereira, G. A. L.; Fontes, A.; Biochim. Biophys. Acta, Gen. Subj. 2018, 1862, 2788.

114. Gerion, D.; Pinaud, F.; Williams, S. C.; Parak, W. J.; Zanchet, D.; Weiss, S.; Alivisatos, A. P.; J. Phys. Chem. B 2001, 105, 8861.

115. Carrillo-Carrión, C.; Moliner-Martínez, Y.; Simonet, B. M.; Valcárcel, M.; Anal. Chem. 2011, 83, 2807.

116. Kim, H.; Jeen, T.; Tran, M. V.; Algar, W. R.; Analyst 2018, 143, 1104.

117. Pathak, S.; Davidson, M. C.; Silva, G. A.; Nano Lett. 2007, 7, 1839.

118. Chouhan, R. S.; Vinayaka, A. C.; Thakur, M. S.; Anal. Bioanal. Chem. 2010, 397, 1467.

119. Prasuhn, D. E.; Deschamps, J. R.; Susumu, K.; Stewart, M. H.; Boeneman, K.; Blanco-Canosa, J. B.; Dawson, P. E.; Medintz, I. L.; Small 2010, 6, 555.

120. Parak, W. J.; Gerion, D.; Zanchet, D.; Woerz, A. S.; Pellegrino, T.; Micheel, C.; Williams, S. C.; Seitz, M.; Bruehl, R. E.; Bryant, Z.; Bustamante, C.; Bertozzi, C. R.; Alivisatos, A. P.; Chem. Mater. 2002, 14, 2113.

121. Hlavata, L.; Striesova, I.; Ignat, T.; Blaskovisova, J.; RuttkayNedecky, B.; Kopel, P.; Adam, V.; Kizek, R.; Labuda, J.; Microchim. Acta 2015, 182, 1715. 
122. Datinská, V.; Klepárník, K.; Belšánová, B.; Minárik, M.; Foret, F.; J. Sep. Sci. 2018, 41, 2961.

123. Voráčová, I.; Klepárník, K.; Lišková, M.; Foret, F.; Electrophoresis 2015, 36, 867.

124. Gupta, S.; Kesarla, R.; Chotai, N.; Misra, A.; Omri, A.; BioMed Res. Int. 2017, 18.

125. Ipe, B. I.; Shukla, A.; Lu, H.; Zou, B.; Rehage, H.; Niemeyer, C. M.; ChemPhysChem 2006, 7, 1112.
126. Lian, S.; Zhang, P.; Gong, P.; Hu, D.; Shi, B.; Zeng, C.; Cai, L.; J. Nanosci. Nanotechnol. 2012, 12, 7703.

Submitted: February 19, 2019

Published online: July 16, 2019 